\title{
Identification and Mapping of Eastern Filbert Blight Resistance Quantitative Trait Loci in European Hazelnut Using Double Digestion Restriction Site Associated DNA Sequencing
}

\author{
Josh A. Honig 1 \\ Department of Plant Biology, School of Environmental and Biological Sciences, Rutgers University, \\ Foran Hall, 59 Dudley Road, New Brunswick, NJ 08901 \\ Megan F. Muehlbauer \\ Rutgers University Cooperative Extension of Hunterdon County, 314 State Route 12, Building 2, \\ Flemington, NJ 08822 \\ John M. Capik, Christine Kubik, and Jennifer N. Vaiciunas \\ Department of Plant Biology, School of Environmental and Biological Sciences, Rutgers University, \\ Foran Hall, 59 Dudley Road, New Brunswick, NJ 08901 \\ Shawn A. Mehlenbacher \\ Department of Horticulture, Oregon State University, 4017 Agricultural and Life Sciences Building, \\ 2750 SW Campus Way, Corvallis, OR 97331 \\ Thomas J. Molnar \\ Department of Plant Biology, School of Environmental and Biological Sciences, Rutgers University, \\ Foran Hall, 59 Dudley Road, New Brunswick, NJ 08901
}

AdDitional InDEX wORDs. ddRADseq, disease resistance, filbert nuts, GBS, genetic linkage map, genotyping-by-sequencing, QTL analysis

\begin{abstract}
European hazelnut (Corylus avellana L.) is an economically important edible nut producing species, which ranked sixth in world tree nut production in 2016. European hazelnut production in the United States is primarily limited to the Willamette Valley of Oregon, and currently nonexistent in the eastern United States because of the presence of a devastating endemic disease, eastern filbert blight (EFB) caused by Anisogramma anomala (Peck) E. Muller. The primary commercial means of control of EFB to date is through the development and planting of genetically resistant european hazelnut cultivars, with an $\boldsymbol{R}$-gene introduced from the obsolete, late-shedding pollinizer 'Gasaway'. Although the 'Gasaway' resistance source provides protection against EFB in the Pacific northwestern United States (PNW), recent reports have shown that it is not effective in parts of the eastern United States. This may be in part because the identification and selection of 'Gasaway' and 'Gasaway'-derived cultivars occurred in an environment (PNW) with limited genetic diversity of $A$. anomala. The objectives of the current research were to develop a genetic linkage map using double digestion restriction site associated DNA sequencing (ddRADseq) and identify quantitative trait loci (QTL) markers associated with EFB resistance from the resistant selection Rutgers H3R07P25 from southern Russia. A mapping population composed of 119 seedling trees was evaluated in a geographic location (New Jersey) where the EFB fungus is endemic, exhibits high disease pressure, and has a high level of genetic diversity. The completed genetic linkage map included a total of 2217 markers and spanned a total genetic distance of $1383.4 \mathrm{cM}$, with an average marker spacing of $0.65 \mathrm{cM}$. A single QTL region associated with EFB resistance from H3R07P25 was located on european hazelnut linkage group (LG) 2 and was responsible for $72.8 \%$ of the phenotypic variation observed in the study. Based on its LG placement, origin, and disease response in the field, this resistance source is different from the 'Gasaway' source located on LG6. The current results, in combination with results from previous research, indicate that the H3R07P25 source is likely exhibiting resistance to a broader range of naturally occurring $A$. anomala isolates. As such, H3R07P25 will be important for the development of new european hazelnut germplasm that combines EFB resistance from multiple sources in a gene pyramiding approach. Identification of EFB resistance in high disease pressure environments representing a diversity of A. anomala populations is likely a requirement for identifying plants expressing durable EFB resistance, which is a precursor to the development of a commercially viable european hazelnut industry in the eastern United States.
\end{abstract}

Received for publication 20 Mar. 2019. Accepted for publication 15 May 2019. This work was partly supported by the Rutgers Center for Turfgrass Science, New Jersey Agricultural Experiment Station, Hatch Act Funds, and the U.S. Department of Agriculture National Institute of Food and Agriculture (Agriculture and Food Research Initiative Competitive Grant 2014-67013-22421 and the Specialty Crops Research Initiative Competitive Grants 2016-04991 and 2009-51181)

${ }^{1}$ Corresponding author. E-mail: honig@sebs.rutgers.edu.
The genus Corylus L. $(2 n=2 x=22)$, in the family Betulaceae, order Fagales, is composed of 11 edible nut producing species (Bassil et al., 2013; Chen et al., 1999; Erdogan and Mehlenbacher, 2000; Muehlbauer et al., 2014a; Yoo and Wen, 2002) that are geographically distributed across North America, Europe, and Asia (Mehlenbacher, 1991). The most commercially important species is the european hazelnut, 
which ranked sixth in world tree nut production in 2016 (743,455 t) [Food and Agricultural Organization of the United Nations (FAO), 2016]. Approximately 10\% of the nuts of this species are sold in-shell, whereas the remaining $90 \%$ are processed as dairy, bakery, chocolate, and confectionery products (Ciarmiello et al., 2014; Petriccione et al., 2010).

The top five leading european hazelnut producers in 2016 included Turkey $(420,000 \mathrm{t})$, Italy (120,572 t), the United States $(34,473 \mathrm{t})$, Azerbaijan (33,941 t), and the Republic of Georgia $(29,500 \mathrm{t})(\mathrm{FAO}, 2016)$. In the United States, commercial european hazelnut production occurs almost exclusively in the Willamette Valley of Oregon, accounting for $99 \%$ of total U.S. production (Chen et al., 2007; Lunde et al., 2000; U.S. Department of Agriculture, 2018). European hazelnut production has been attempted in the eastern United States as far back as the colonial time period (Thompson et al., 1996); however, the presence of a devastating endemic disease, EFB caused by the fungus $A$. anomala, harbored by the tolerant native american hazelnut (Corylus americana Marsh.), has prevented the establishment of a viable european hazelnut industry east of the Rocky Mountains (Capik and Molnar, 2012, 2014; Fuller, 1908; Halsted, 1892; Johnson and Pinkerton, 2002; Thompson et al., 1996).

European hazelnut production in the PNW United States remained EFB free from the time of the establishment of the industry in the late 1800s (Thompson et al., 1996) until an inadvertent introduction of $A$. anomala occurred in southwestern Washington in the 1960s (Davison and Davidson, 1973). Since that time, EFB has spread throughout the Willamette Valley of Oregon (Johnson and Pinkerton, 2002; Pinkerton et al., 1992). Disease management practices now include scouting for cankers, extensive pruning, and liberal fungicide applications; however, they are not always completely effective, which is a challenge when dealing with a perennial stem canker disease that has a 16-month latent period. This point, combined with the expense associated with these control measures, indicates that host genetic resistance is the most sustainable, long-term, economically viable solution for EFB management (Chen et al., 2007; Johnson et al., 1996; Julian et al., 2008; Lunde et al., 2006; Mehlenbacher, 1995; Molnar et al., 2013; Sathuvalli et al., 2010).

The first source of host genetic resistance identified in the PNW was the obsolete, late-shedding pollinizer $C$. avellana 'Gasaway' (Cameron, 1976; Mehlenbacher et al., 1991). Although 'Gasaway' exhibits low yields and poor nut and kernel characteristics, when this cultivar is crossed with EFBsusceptible germplasm, it transmits resistance to its progeny in a 1:1 resistant:susceptible ratio, indicative of carrying a dominant EFB resistance allele ( $R$-gene) in a heterozygous state at a single locus (Coyne et al., 1998; Mehlenbacher et al., 1991, 2006; Osterbauer et al., 1997; Sathuvalli et al., 2017). 'Gasaway' has subsequently been widely used in the Oregon State University (OSU) european hazelnut breeding program, resulting in the release of many advanced generation european hazelnut cultivars (Mehlenbacher et al., 2009, 2011, 2013, 2014, 2016) and pollinizers (Mehlenbacher and Smith, 2004; Mehlenbacher and Thompson, 1991; Mehlenbacher et al., 2012). Largely based on these new EFB-resistant cultivars, the Oregon european hazelnut industry expanded to $31,809.5$ ha in 2018 (N. Wiman, personal communication).

Hazelnut breeders and plant pathologists have been concerned about the long-term durability of, and reliance on, a single source of EFB resistance in the european hazelnut industry (Coyne et al., 1998; Leadbetter et al., 2016; Lunde et al., 2006; Molnar et al., 2010a; Osterbauer, 1996; Pinkerton et al., 1998). Adding to this concern is that $A$. anomala found in the PNW is believed to have originated and spread from a single point introduction (Gottwald and Cameron, 1980; Johnson et al., 1996; Pinkerton et al., 1993), raising the possibility that the 'Gasaway' $R$-gene may have been initially discovered and screened against a limited number of fungal isolates. Support for this hypothesis can be found in recent research using microsatellite or simple sequence repeat (SSR) markers to study the diversity of $A$. anomala samples collected from multiple locations across the United States. Studies showed that $A$. anomala isolates collected from Oregon were genetically similar, whereas isolates collected from east of the Rocky Mountains were found to be much more genetically diverse (Cai et al., 2013; Muehlbauer et al., 2014b, 2019; Tobia et al., 2017). In addition, 'Gasaway' and its offspring, which have been shown to be resistant to EFB in Oregon [and were initially resistant to EFB in New Jersey (T.J. Molnar, unpublished data)], have recently been shown to be susceptible to EFB at Rutgers University in New Jersey (Capik and Molnar, 2012; Capik et al., 2013; Molnar et al., 2010a, 2010b; T.J. Molnar, unpublished data). This is a location where the fungus is endemic, exhibits greater genetic diversity (Cai et al., 2013; Muehlbauer et al., 2014b, 2019), and more important, has also been shown to exhibit pathogenic variation (Molnar et al., 2010a; T.J. Molnar, unpublished data).

In response to the concerns and observations listed previously, both the OSU and Rutgers University hazelnut breeding programs have outlined goals to collect, identify, and develop $C$. avellana germplasm sources with EFB resistance that differ from the 'Gasaway' $R$-gene source. This has included the goals of searching for $R$-genes exhibiting resistance to multiple isolates of $A$. anomala, and/or the identification or development of $C$. avellana germplasm with multiple $R$-genes in a gene pyramiding approach. Starting in 2002, a wide germplasm collection was made across the native range of $C$. avellana in eastern Europe and the Caucasus Republics. In total, more than 5000 seedling trees were grown from nearly 200 individual seed lots collected across 10 countries. After 16 years of evaluation in New Jersey, $\approx 3 \%$ of this collected germplasm has remained resistant to or highly tolerant of EFB (Capik et al., 2013; Leadbetter et al., 2016; Molnar et al., 2007, 2018), whereas the 'Gasaway' source has not offered the same level of protection (Capik and Molnar, 2012; Capik et al., 2013; Molnar et al., 2010a, 2010b; Muehlbauer et al., 2018; T.J. Molnar, unpublished data) and has shown differential pathogenic responses to $A$. anomala isolates in New Jersey and elsewhere across the native range of the fungus (Molnar et al., 2010a; T.J. Molnar, unpublished data). These observations and the wide origins of the plant materials (Muehlbauer et al., 2014a), when considered together, suggest that some of the newly identified EFB-resistant germplasm may not only differ from the 'Gasaway' source but also provide resistance to a broader range of $A$. anomala isolates.

The $C$. avellana reference genetic linkage map and trait association research at OSU have shown that the putative 'Gasaway' $R$-gene resides on european hazelnut LG6 (Colburn et al., 2015, 2017; Gürcan and Mehlenbacher, 2010; Mehlenbacher et al., 2006; Sathuvalli and Mehlenbacher, 2013; Sathuvalli et al., 2012, 2017). Interestingly, related work at 
OSU showed that resistance from the Spanish cultivar Culplà, the Serbian cultivar Crvenje, and OSU 495.072 (a seed source from the N.I. Vavilov Research Institute of Plant Industry in St. Petersburg, Russia) also map to LG6 in a region similar to the 'Gasaway' source (Colburn et al., 2015). However, resistance from the Spanish cultivar Ratoli was shown to reside on LG7 (Sathuvalli et al., 2011a), as does resistance from C. americana 'Rush' (Bhattarai et al., 2017), whereas resistance from $C$. avellana OSU 759.010 (a selection from the Republic of Georgia) resides on LG2 (Sathuvalli et al., 2011b). All of these genetic mapping and QTL identification studies were conducted in the PNW (Oregon), where the genetic diversity of $A$. anomala appears to be limited. The objective of the current research was as follows: 1) conduct ddRADseq-based genetic linkage mapping and QTL experiments in a cross of european hazelnut selection Rutgers H3R07P25, resistant to EFB in the eastern United States, and EFB-susceptible selection OSU 1155.009 ; 2) expose full-sib progeny from that cross to naturally occurring high EFB disease pressure in New Jersey where $A$. anomala is endemic and genetically diverse; and 3 ) compare the LG locations of newly identified EFB resistance QTLs to those previously reported.

\section{Materials and Methods}

\section{Plant Material}

Mapping POPUlation Parents. The full-sib mapping population used in the current study was developed from a controlled cross between two heterozygous individuals, with EFB-resistant H3R07P25 as the female parent and EFBsusceptible OSU 1155.009 as the male parent. H3R07P25 originated as a single EFB-resistant selection from a population of trees (seed lot RUS 12) grown from open-pollinated seed collected in Aug. 2002 at an outdoor market in Holmskij, Krasnodarskiy Kray, Russia (southern Russia, Black Sea region) (Capik et al., 2013; Molnar et al., 2007). The initial EFB resistance screening and identification of H3R07P25 was described in detail in Molnar et al. (2007). H3R07P25 was one of only 13 trees of 605 described in Molnar et al. (2007) that remained free of EFB. In addition, the original H3R07P25, as well as three grafted trees of the same clone, have been continually exposed to EFB in additional field trials at Rutgers, where all have remained disease free as of Dec. 2018 (Capik et al., 2013; Leadbetter et al., 2016; T.J. Molnar, unpublished data). H3R07P25 also remained free of EFB after greenhouse exposure in Oregon as well as in the field (S.A. Mehlenbacher, unpublished data). The male parent, OSU 1155.009, was identified as EFB-susceptible at OSU (data not shown) and selected as a parent for the cross based on its susceptibly, improved nut and kernel characteristics, and high yields. It originated from a cross of OSU 474.084 ('Lewis' $\times$ 'Tonda di Giffoni') $\times$ OSU 540.084 (a full sibling of 'Sacajawea') (Mehlenbacher et al., 2000, 2008).

MAPPING POPUlation DEVELOPMENT AND FIELd EVALUATION OF EFB RESPONSE. The controlled hybridization $(\mathrm{H} 3 \mathrm{R} 07 \mathrm{P} 25 \times$ OSU 1155.009) was conducted following protocols described in Mehlenbacher (1994). H3R07P25 was located at Rutgers University Horticultural Research Farm \#3 (East Brunswick, NJ) and pollen of OSU 1155.009 was provided by OSU. Pollen was collected in Jan. 2011 and stored at $-28.9^{\circ} \mathrm{C}$ until shipping on dry ice by overnight mail before its use in Feb. 2011 at Rutgers University. In Sept. 2011, the resulting hybrid seeds were harvested, provided a moist chilling period at $4{ }^{\circ} \mathrm{C}$ for 4 months, then germinated and grown in the greenhouse according to protocols described by Molnar and Capik (2012). Seedlings were removed from the greenhouse in June 2012 for acclimation outdoors under shadecloth (40\% shade) until field planting in Nov. 2012 at Rutgers University Horticultural Research Farm \#3. Tree spacing was $1.0 \mathrm{~m}$ in the row by $3.0 \mathrm{~m}$ between rows. Weed control using herbicides, irrigation, and fertilizer application were provided as needed, with no use of insecticides or fungicides. Trees were field inoculated by tying diseased twigs to the stems of the seedlings before budbreak each year (Molnar et al., 2007) to keep disease pressure high and reduce the possibility of susceptible plants escaping infection. In addition, EFB infected trees in nearby research plots contributed a steady influx of additional EFB inoculum. All of the trees were evaluated in Jan. 2016 and Jan. 2017 for response to EFB, which was recorded using a scale of 0 to 5 , according to a modified index adapted from Pinkerton et al. (1992): $0=$ no detectable EFB (includes presence of "sunken lesion" phenotypes where a few small sunken cankers that lack fungal stromata) $=0 \%$ of stems diseased; $1=$ single canker (with fully formed stromata $) \cong 1 \%$ of stems diseased; $2=$ multiple cankers on a single branch $\cong 5 \%$ of stems diseased; $3=$ multiple branches with cankers $\cong 25 \%$ of stems diseased; $4=50 \%$ branches with cankers $\cong 50 \%$ of stems diseased; $5=$ all branches contain cankers (except basal sprouts) $=100 \%$ of stems diseased. Plants scored 0 or 1 were considered resistant to infection by $A$. anomala. The 0 to 5 scale was converted to percent disease, as described previously, for the QTL analyses.

DNA EXTRACTION. Genomic DNA was isolated from both parents and 119 seedling samples using a DNeasy Plant Mini Kit (Qiagen, Germantown, MD), following the manufacturer's instructions. Sample DNA quality and quantity were assessed using a spectrophotometer (NanoDrop; Thermo Fisher Scientific, Waltham, MA). Extracted DNA was used for SSR marker characterization and the preparation of ddRADseq libraries for all samples.

Microsatellite (SSR) MARKers. A total of 126 microsatellite or SSR markers from various sources (Akin et al., 2016; Bassil et al., 2005; Bhattarai and Mehlenbacher, 2018; Boccacci et al., 2005; Colburn et al., 2015, 2017; Gürcan and Mehlenbacher, 2010; Gürcan et al., 2010a, 2010b; Ives et al., 2014; Mehlenbacher et al., 2006; Sathuvalli and Mehlenbacher, 2013; Sathuvalli et al., 2012) were genotyped as potential anchor markers for LG comparison between the current genetic linkage map and previous maps (Beltramo et al., 2016; Bhattarai and Mehlenbacher, 2017; Colburn et al., 2015, 2017; Gürcan et al., 2010a; Ives et al., 2014; Mehlenbacher et al., 2006; Sathuvalli et al., 2011a, 2011b, 2012; Torello Marinoni et al., 2018). A prefix indicated that LG was added to the SSR marker names. Polymerase chain reaction (PCR) amplification of SSR markers for genotyping was conducted in $13-\mu \mathrm{L}$ reactions, using $\approx 5 \mathrm{ng}$ genomic DNA per sample, $1 \times$ PCR buffer, $2.0 \mathrm{~mm} \mathrm{MgCl}_{2}, 0.25 \mathrm{~mm}$ dNTPs, 0.5 pmol forward primer with 5'-M13(-21) addition (Schuelke, 2000), 1 pmol reverse primer with 5'-"PIG-tailing', addition (Brownstein et al., 1996), 1 pmol forward M13(-21) primer with FAM, NED, PET, or VIC fluorescent labels (Schuelke, 2000), and 0.5 U IMMOLASE DNA polymerase (Bioline; Meridian Life Science, Memphis, TN). All SSR marker primers were synthesized by Integrated DNA Technologies (Coralville, IA). Thermalcycling (9700 GeneAmp PCR system; Applied Biosystems, 
Foster City, CA) was performed under the following conditions: initial denaturation of $94{ }^{\circ} \mathrm{C}$ for $5 \mathrm{~min} ; 30$ cycles of $94{ }^{\circ} \mathrm{C}$ for $30 \mathrm{~s}, 55^{\circ} \mathrm{C}$ for $45 \mathrm{~s}, 72{ }^{\circ} \mathrm{C}$ for $45 \mathrm{~s} ; 20$ cycles of $94^{\circ} \mathrm{C}$ for 30 $\mathrm{s}, 53{ }^{\circ} \mathrm{C}$ for $45 \mathrm{~s}, 72{ }^{\circ} \mathrm{C}$ for $45 \mathrm{~s}$; with a final extension of $72{ }^{\circ} \mathrm{C}$ for $10 \mathrm{~min}$. PCR products were analyzed using capillary electrophoresis (3500xl Genetic Analyzer, Applied Biosystems), and sized using LIZ 600 size standard v2.0 (Applied Biosystems) and Genemapper 5.0 software (Applied Biosystems).

DDRADSEQ LIBRARY CONSTRUCTION AND ILLUMINA SEQUENCING. ddRADseq libraries for H3R07P25, OSU 1155.009, and 119 mapping population seedlings were created using a protocol adapted from Poland et al. (2012). Briefly, 200 ng of genomic DNA from each individual sample was double digested with the rare-cutting PstI (NEB, Ipswich, MA) and the common-cutting $M s p I$ restriction enzymes for $2 \mathrm{~h}$ at $37^{\circ} \mathrm{C}$. Uniquely barcoded [5-10 base pair (bp)] forward PstI adapters and reverse $M s p I$ Y-adapters were ligated to the digested european hazelnut DNA in a mastermix consisting of $200 \mathrm{U}$ of T4 DNA ligase, $2 \mu \mathrm{L}$ of $10 \times$ NEBuffer 4 , and $4 \mu \mathrm{L}$ of ATP $(10 \mathrm{~mm})(\mathrm{NEB}$, Ipswich, MA) per sample. The ligation reaction was incubated for $2 \mathrm{~h}$ at $22{ }^{\circ} \mathrm{C}$, with ligase inactivation occurring via an additional incubation of $20 \mathrm{~min}$ at $65^{\circ} \mathrm{C}$. Samples were then subjected to a "clean-up" with $0.5 \mathrm{v} / \mathrm{v}$ magnetic beads (Agencourt Ampure XP; Beckman Coulter, Brea, CA), followed by washing with $70 \%$ ethanol to remove DNA fragments smaller than $300 \mathrm{bp}$. Individual cleaned library samples were then PCR amplified, with primers that included sequences allowing for Illumina (San Diego, CA) next-generation sequencing (NGS) flow cell binding, under the following thermalcycling conditions: initial denaturation of $95{ }^{\circ} \mathrm{C}$ for 30 $\mathrm{s}$; followed by 16 cycles of $95^{\circ} \mathrm{C}$ for $30 \mathrm{~s}, 62^{\circ} \mathrm{C}$ for $20 \mathrm{~s}, 68^{\circ} \mathrm{C}$ for $15 \mathrm{~s}$; with a final extension of $68{ }^{\circ} \mathrm{C}$ for $5 \mathrm{~min}$. All DNA libraries were then quantified using a fluorometer (Qubit 3.0; Thermo Fisher Scientific), normalized to $5 \mathrm{ng} \cdot \mu \mathrm{L}^{-1}$, and then pooled for Illumina short-read sequencing. Before sequencing, pooled libraries were subjected to a second clean-up with magnetic beads and wash step as previously described. Final pooled library quality was assessed via high-resolution automated electrophoresis (2100 Bioanalyzer System; Agilent Technologies, Santa Clara, CA).

Sequencing pools were constructed such that all progeny samples were divided equally across six MiSeq runs (Illumina) $(2 \times 300$ paired-end) conducted at Rutgers University (New Brunswick, NJ), as well as an additional single pool of all samples on a single lane of a HiSeq 2500 [Illumina $(2 \times 125$ paired-end high-output sequencing)] sequenced by Genewiz, Inc. (South Plainfield, NJ). Each mapping population parent sample was sequenced a total of 14 times across all sequencing runs. Each sequencing run was loaded with $10 \%$ and $30 \%$ PhiX for the MiSeq and HiSeq sequencing runs, respectively.

DDRADSEQ SINGLE NUCLEOTIDE POLYMORPHISM (SNP) MARKER CALLING. Raw ddRADseq FASTQ data files were processed using the software pipeline Stacks 1.30 (Catchen et al., 2011, 2013). All data files were demultiplexed according to barcode, quality filtered, and trimmed to $100 \mathrm{bp}$ using the process_radtags command (with barcode rescue, $-r$ ). The denovo_map.pl command wrapper of Stacks was then used for de novo SNP marker discovery (without reference genome), specifying the "CP" genetic map (- A). denovo_map.pl combines the ustacks, cstacks, and sstacks programs. Briefly, the ustacks program was used to align matching reads of all samples into putative loci (stacks) and for making SNP marker calls, with a minimum of four matching reads $(-\mathrm{m})$ required to create a stack and a maximum distance (nucleotide mismatch, - M) of three. A catalog of loci was then generated from the two parental genotypes using the cstacks program, followed by matching the generated stacks of the progeny samples back to the parental catalog using the sstacks program. Finally, the genotypes program of Stacks was used to export map-specific genotypes in a format compatible with JoinMap 4.1 (van Ooijen, 2006) and OneMap/R (Margarido et al., 2007). Loci with more than $4.2 \%$ missing data (i.e., loci must be present in 115 progeny) were excluded from further analysis ( $\mathrm{r}$ of genotypes program).

LinKAGE MAP CONSTRUCTION. Three approaches were used for the construction of genetic linkage maps using the software JoinMap 4.1 and the package OneMap/R. The first strategy used the two-way pseudo-testcross approach for cross-pollinated (CP) species (Grattapaglia and Sederoff, 1994) in JoinMap 4.1, with additional procedures as described by Mehlenbacher et al. (2006). Briefly, both SSR and SNP markers were initially combined into one dataset and formatted so that markers were coded as heterozygous in one parent and homozygous in the other parent $(l m \times l l$ or $n n \times n p)$. All markers were then recoded as BC1 markers in JoinMap 4.1, and initial maps for each parent were generated. Markers assumed to be linked in repulsion, and not initially mapped, were recoded as "dummy variables" (a for present, and $\mathrm{h}$ for absent) to allow for mapping with markers previously assumed to be in coupling phase to create a single map for each parent. All individual markers were subjected to $\chi^{2}$ analysis in JoinMap to test for goodness of fit to expected segregation ratios (1:1), and markers showing severe segregation distortion $(P \leq 0.001)$ were excluded from further analyses. Marker grouping was performed over a range of logarithm of odds (LOD) values from 2 to 30, with a step of one. After grouping, the order of loci was determined using the multipoint maximum likelihood mapping algorithm with a Gibbs sampling procedure (van Ooijen, 2006). This algorithm specifically deals with deterioration in computation time of the regression mapping algorithm in high-density linkage maps with greater than an average of 50 loci per LG (van Ooijen, 2006). Spatial sampling was conducted with five threshold values $(0.1,0.05,0.03,0.02$, and 0.01$)$, with three map optimization runs for each spatial sampling. Map optimization parameters included the following: chain length of 1000; cooling control parameter of 0.001 ; and chain termination after 10,000 chains without improvement. Map distances were converted from recombination frequency to centimorgans using the Kosambi mapping function.

The second strategy, also in JoinMap 4.1, used the extension of the multipoint maximum likelihood mapping algorithm to CP full-sib populations described in van Ooijen (2011a). In this extension, the JoinMap 4.1 software first constructs two parental maps simultaneously, estimating phasing, and then integrates the two parental maps into a final single map (van Ooijen, 2011b). For this strategy, SSR and SNP markers were combined into one dataset, with both common markers $(a b \times$ $c d$, $e f \times e g$, and $h k \times h k)$ and parental markers $(l m \times l l$ and $n n \times$ $n p$ ) represented. Marker grouping was initially performed over a range of independence LOD values from 2 to 30, with a step of one; with a final LOD value $\geq 24$ used to group loci. After grouping, the multipoint maximum likelihood mapping algorithm was used to determine the order of loci, with the same 
parameters described previously. The Kosambi mapping function was used to determine map distances (centimorgans), also as described previously.

The third strategy was implemented in the package One$\mathrm{Map} / \mathrm{R}$. The core functionality of this package is the construction of integrated linkage maps combining information from markers exhibiting mixed segregation patterns (common and parental markers), as well as simultaneous maximum likelihood estimation of linkage and linkage phases between markers in outcrossing (CP) species (Margarido et al., 2007). Briefly, locus genotypes used in the previous JoinMap analyses were imported into OneMap/R in outcross format using the read.outcross function. Next, the recombination fractions among all pairs of markers was calculated using the rf.2pts function. Markers were assigned to LGs using the group function, testing a range of LOD scores (5-30) and a range of maximum recombination frequencies (0.2-0.5). A final LOD score of LOD $=20$ was used to create all LGs. The ordering of markers within each group was determined using the order.seq function, with an initial comparison of six informative markers, addition of markers sequentially, and an LOD threshold of three. All LG map distances (centimorgans) were calculated using the Kosambi mapping function. Alternative marker orders on all LGs were tested with the ripple.seq function. Maps from all three genetic mapping approaches described previously were viewed using MapChart 2.30 (Voorips, 2002).

QTL ANALYSIS. QTL analysis of single year (2016 and 2017) and mean EFB response was conducted using MapQTL 6.0 software (van Ooijen, 2009). Because of memory constraints with the CP mapping population, the two-way pseudo-testcross approach with markers recoded as $\mathrm{DH}$ population type as described by van Ooijen (2009) was used to construct two separate parental maps that were then used for all QTL mapping procedures (Herrmann et al., 2006; McAdam et al., 2013; Studer et al., 2006; van Heerden et al., 2014; Yun et al., 2014; Zyprian et al., 2016). Initial analysis was conducted using interval mapping (IM), followed by multiple rounds of multiple QTL mapping (MQM) to refine the location and magnitude of QTL. Briefly, putative QTL identified from IM were used as initial cofactors in the MQM mapping analysis. For MQM mapping, a backward elimination procedure was then used to select additional cofactors in subsequent rounds of MQM, until QTL positions were stabilized. LOD thresholds for QTL significance were determined by permutation test $(n=1000)$, with a genome-wide significance level of 0.05 . QTL positions and magnitude were visualized using MapChart 2.30 (Voorips, 2002).

\section{Results}

Segregation for EFB Resistance. The EFB response of the 119 seedlings from the cross between H3R07P25 and OSU 1155.009 was 55 resistant and 64 susceptible (Table 1). EFB ratings were similar across years (data not shown), and were therefore averaged for $\chi^{2}$ analysis. The segregation pattern fits the expected ratio of one resistant to one susceptible, indicating that EFB resistance from H3R07P25 may be controlled by a dominant allele at a single locus in a heterozygous state.

Microsatellite (SSR) Genotyping. Ninety SSR markers previously used for genotyping hazelnuts and linkage mapping (Beltramo et al., 2016; Bhattarai and Mehlenbacher, 2017; Colburn et al., 2015, 2017; Gürcan et al., 2010a; Ives et al.,
Table 1. Segregation for response to eastern filbert blight (EFB) in 119 european hazelnut seedlings from a cross of EFB-resistant Rutgers H3R07P25 × EFB-susceptible Oregon State University (OSU) 1155.009 .

\begin{tabular}{lcccccc}
\hline & \multicolumn{3}{c}{ Seedlings (no.) } & & \multicolumn{3}{c}{$\chi^{2}$} \\
\cline { 2 - 3 } \cline { 5 - 7 } Parents & Resistant & Susceptible & & Test ratio & Value & $P$ \\
\hline H3R07P25 & 55 & 64 & & $1: 1$ & 0.54 & 0.47 \\
$\quad$ OSU 1155.009 & & & & &
\end{tabular}

${ }^{\mathrm{z}}$ Plants with an average rating of 0 to 1.5 for the combined 2016 and 2017 seasons were considered resistant to EFB. Plants with an average rating score of 3.5 to 5.0 were considered susceptible. There were no plants with an average rating score of 1.6 to 3.4 . Rating scale adapted from Pinkerton et al. (1992).

2014; Mehlenbacher et al., 2006; Sathuvalli et al., 2011a, 2011b, 2012; Torello Marinoni et al., 2018) were found to be polymorphic between the two parents of the mapping population cross and are listed along with their motif type and primer sequence information (Supplemental Table 1). Six of the 90 SSR markers were removed from the final genetic linkage map because of greater than $4.2 \%$ missing data in the progeny population or suspect linkages. Thirty-one of the remaining SSR markers segregated 1:1 $(l m \times l l$ or $n n \times n p)$; 50 SSR markers segregated 1:1:1:1 $(a b \times c d$ or $e f \times e g)$; whereas three SSR markers segregated 1:2:1 $(h k \times h k)$ (Supplemental Table 1).

SNP MARKER DISCOVERY AND POLYMORPHIC LOCI DEVELOPMENT. The summary data for the sequencing runs performed in this study are presented in Supplemental Table 2. Each ddRADseq sequencing library was sequenced individually in a single lane of either an MiSeq or HiSeq instrument platform. The mean $\mathrm{Q}$ scores for the MiSeq sequencing runs ranged from 32.4 to 34.3 , with an average mean $Q$ score of 33.7 across all six MiSeq sequencing runs. The mean Q score for the single HiSeq 2500 sequencing run was 29.0. The percentage of $\mathrm{Q}$ score $\geq \mathrm{Q} 30$ for the MiSeq sequencing runs ranged from $76.9 \%$ to $84.9 \%$, with an average percentage of Q score $\geq$ Q30 of $82.3 \%$ for all six MiSeq sequencing runs, whereas the single HiSeq 2500 sequencing run had a percentage of $Q$ score $\geq Q 30$ of $63.5 \%$.

The combined MiSeq and HiSeq 2500 sequencing runs yielded a total of $101.11 \mathrm{~Gb}$ of DNA sequences (Supplemental Table 2). After demultiplexing and trimming to remove barcodes, adapters, and low-quality bases (at sequence fragment ends) (Stacks process_radtags), the number of sequencing reads per seedling sample ranged from a low of slightly more than 1.83 million reads to a high of slightly more than 4.06 million reads, with an average of 2.89 million reads across all 119 samples. The parental samples had slightly more than 14.03 million and 15.76 million reads for OSU 1155.009 and H3R07P25, respectively. This resulted in an average of $\cong 5.2$ $\times$ number of reads for the parental samples relative to the seedling samples, where a substantial parental read number excess is important for generating accurate SNP marker calls in the Stacks software pipeline. Raw reads for the two parental samples and all 119 seedling samples were deposited in the National Center for Biotechnology Information Sequence Read Archive database (PRJNA542160) (Leinonen et al., 2011).

The Stacks software retained read catalog for the mapping population parents and progeny (after the ustacks, cstacks, and sstacks steps) contained an average of 110,233 stacks, 16,741 polymorphic loci, and 25,864 raw SNP marker calls. After 
further filtering for progeny individuals containing $<4.2 \%$ missing data, 2894 SNP markers were retained. An additional 761 SNP markers were removed from the dataset before final linkage grouping due to duplicated loci, suspect linkages, failure to group during linkage mapping, and severe segregation distortion ( $P \leq 0.001)$. A total of 1927 of the remaining 2133 SNP markers segregated 1:1 $(l m \times l l$ or $n n \times n p)$ in the progeny population; 18 SNP markers segregated 1:1:1:1 $(a b \times c d$ or $e f \times$ eg); whereas 188 SNP markers segregated 1:2:1 $(h k \times h k)$ (Supplemental Table 3).

LiNKAGE MAP CONSTRUCTION. The three previously described approaches used for linkage mapping (two-way pseudo-testcross in JoinMap 4.1; integrated linkage map in JoinMap 4.1; and integrated map in OneMap/R) of the 2133 SNP markers and 84 SSR markers produced identical results for the grouping analyses, with only very minor differences in microcolinearity between marker positions. Therefore, only the integrated linkage map from JoinMap 4.1 is presented in Fig. 1 and Supplemental Figs. 1-11. At LOD $\geq 24$, JoinMap 4.1 returned 11 integrated LGs, corresponding to the expected haploid chromosome number for european hazelnut $(n=x=11)$ (Fig. 1, Supplemental Figs. 1-11) (Mehlenbacher et al., 2006). The map included a total of 2217 markers and spanned a total genetic distance of $1383.4 \mathrm{cM}$ with an average marker spacing of $0.65 \mathrm{cM}$. The genetic distance of individual LGs ranged from a low of $70.5 \mathrm{cM}$ (98 markers) for LG3, to a high of 196.0 cM (269 markers) for LG2. LG assignments were made via syntenic comparisons of SSR anchor markers to previous european hazelnut linkage maps (Beltramo et al., 2016; Bhattarai and Mehlenbacher, 2017; Colburn et al., 2015, 2017; Gürcan and Mehlenbacher, 2010; Gürcan et al., 2010a; Ives et al., 2014; Mehlenbacher et al., 2006; Sathuvalli and Mehlenbacher, 2013; Sathuvalli et al., 2011a, 2011b, 2012;

Torello Marinoni et al., 2018). Of the remaining 84 polymorphic SSR markers in the current linkage map, two SSR markers (B608 and B635) were not previously assigned to LGs on previous OSU european hazelnut linkage maps. For the remaining 82 polymorphic SSR markers, two SSR markers (B640 and B773) mapped to a different $L G$ on the current linkage map, compared with LG locations on previous OSU european hazelnut linkage maps. Interestingly, the placement of B640 on LG4 in the current linkage map was similar to its placement on the map reported by Beltramo et al. (2016) (also LG4). The 80 remaining SSR markers were then used as anchor markers for LG assignment, with a high of 11 anchored SSR markers on LG9, a low of five anchored SSR markers on LG10, and an average of 7.3 anchored SSR markers per LG (Supplemental Table 1, Supplemental Figs. 1-11).

QTL ANALYSIS. The initial IM QTL analysis in MapQTL 6.0 identified three potential marker/trait (EFB response) association regions on LG2, LG4, and LG9, all associated with the EFB-resistant H3R07P25 parental map [using the DH two-way pseudo-testcross approach for QTL mapping (van Ooijen, 2009)]. Further refinement of QTL location using cofactor selection and MQM mapping resulted in identification of a single EFB resistance QTL region on LG2 associated with two ddRADseq SNP markers (RUCaS1_18603 and RUCaS1_14004) (Fig. 2). QTL LOD scores for RUCaS1_18603 and RUCaS1_14004 were 32.5 and 34.8, respectively (Fig. 2), whereas the average percent phenotypic variation (EFB disease response) explained by the QTL region spanning the two SNP markers was $72.8 \%$, indicating a major QTL region associated with EFB disease resistance. The initial IM marker/trait association regions on LG4 and LG9 fell far below the permutation test-calculated LOD threshold of 3.0 during the cofactor selection and MQM mapping analyses steps. BLASTN results for the sequences containing the RUCaS1_18603 and RUCaS1_

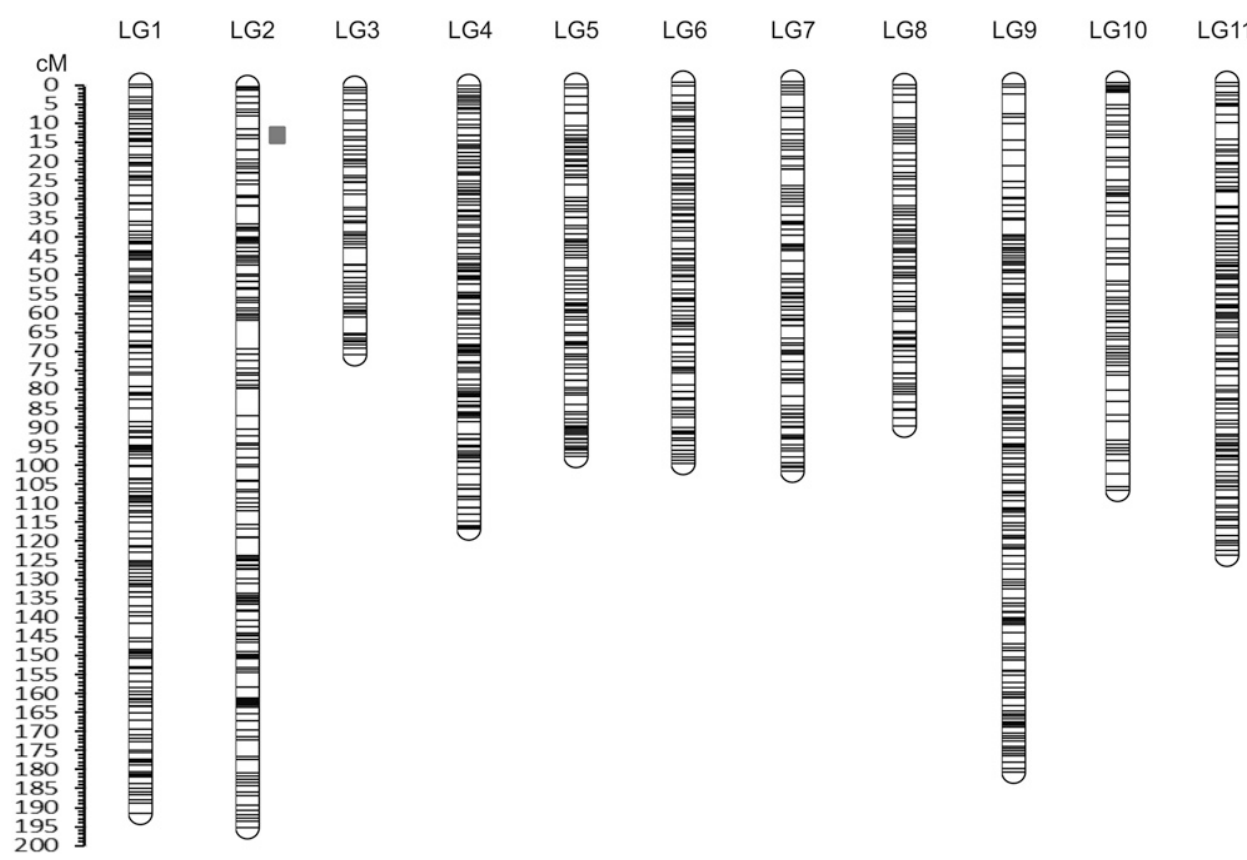

Fig. 1. A genetic linkage map constructed from 119 european hazelnut seedlings from a cross of Rutgers H3R07P25 × Oregon State University (OSU) 1155.009 showing eastern filbert blight (EFB) disease resistance quantitative trait loci (QTL) region (gray box) on linkage group (LG) 2. Genetic distance (Kosambi) is reported in centimorgans.
14004 SNP markers (Supplemental Table 3) returned no matches (data not shown).

\section{Discussion}

Before the advent of NGS technologies, genetic linkage mapping and QTL identification experiments were often conducted with DNA markers such as restriction fragment length polymorphism markers, random amplified polymorphic DNA (RAPD) markers, amplified fragment length polymorphism markers, microsatellite or SSR markers, or some combination thereof. Although many of these markers proved valuable (and are still valuable for anchoring between linkage maps), the high costs, low throughput, and laborious nature of working with these marker systems resulted in low coverage linkage maps requiring an extensive investment of time and resources to 


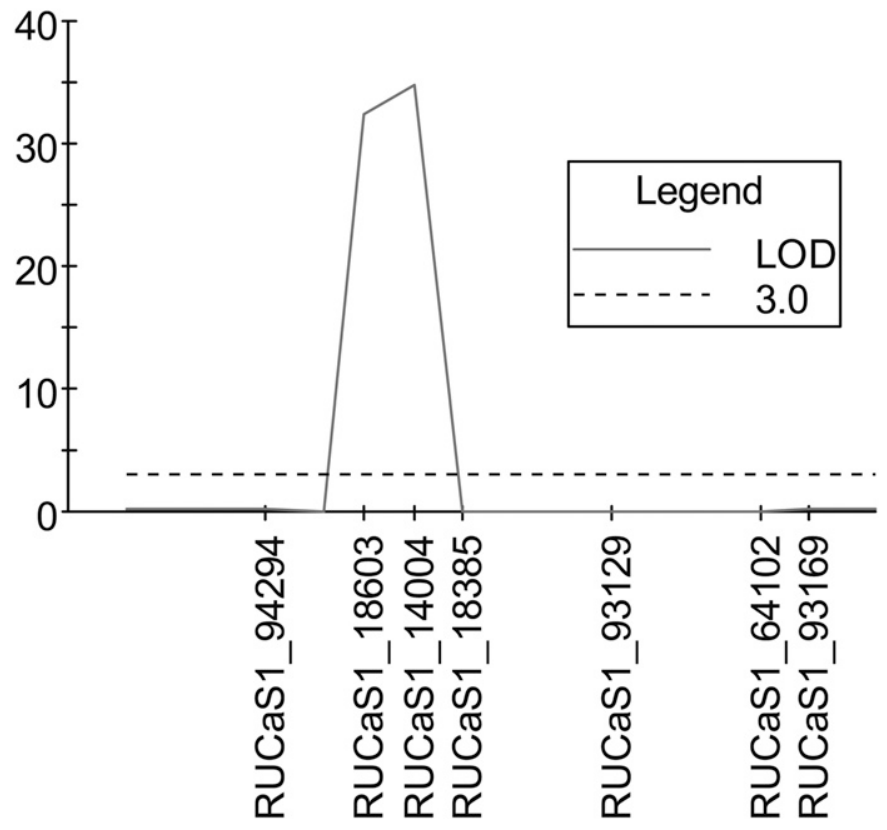

Fig. 2. The quantitative trait locus (QTL) on the genetic linkage map of the european hazelnut cross Rutgers H3R07P25 × Oregon State University 1155.009 from MapQTL6. The eastern filbert blight (EFB) disease resistance QTL and single nucleotide polymorphism (SNP) markers RUCaS1_14004 and RUCaS1_18603 are located on linkage group (LG) 2. Logarithm of odds (LOD) thresholds for QTL significance (dashed line) were determined by permutation test $(n=1000)$, with a genome-wide significance level of 0.05 . QTL LOD scores (solid line) for RUCaS1_18603 and RUCaS1_14004 were 32.5 and 34.8, respectively. The average percent phenotypic variation (EFB disease response) explained by the QTL region spanning the two SNP markers was $72.8 \%$.

construct. In contrast, reduced representation sequencing techniques, such as genotyping-by-sequencing or ddRADseq, that use the massively parallel sequencing throughput of NGS systems, are a relatively straightforward, highly specific, highly reproducible, and economic means of generating thousands to tens of thousands of SNP markers in a short period (Elshire et al., 2011; Poland et al., 2012). This high-throughput and lower cost methodology can then be used to quickly generate genetic linkage maps and identify marker trait associations to answer a broader range of research questions than have traditionally been investigated in the past. In this study, a SNP marker-based ddRADseq genetic linkage map of european hazelnut was created with relatively dense genome coverage similar to other recently developed SNP marker based linkage maps of european hazelnut (Rowley et al., 2018; Torello Marinoni et al., 2018), and then QTL experiments were conducted to specifically determine whether novel or previously known source(s) of EFB resistance QTL were associated with the disease resistance phenotype observed in an environment with high EFB disease pressure (New Jersey location).

The putative 'Gasaway' $R$-gene (as well as resistance from 'Culplà', 'Crvenje', 'Uebov', OSU 408.040, and OSU 495.072) was previously shown to reside on european hazelnut LG6 (Colburn et al., 2015, 2017; Gürcan and Mehlenbacher, 2010; Mehlenbacher et al., 2006; Sathuvalli and Mehlenbacher, 2013; Sathuvalli et al., 2012). Interestingly, EFB resistance from
H3R07P25 resides on LG2 (Fig. 1, Supplemental Fig. 2). An important finding from this work is that the resistance source from H3R07P25 is different from the 'Gasaway' resistance source (previously found to reside on LG6). This is based on the differences in LG locations of resistance QTL (and lack of a resistance signal on LG6 in the current work), as well as the phenotype of the plants protected by this source of resistance in a high disease pressure environment within the native range of the fungus. The position of the disease resistance locus on LG2 in H3R07P25 appears to be similar to the disease resistance source identified in OSU 759.010 (selection from the Republic of Georgia), as both QTL locations are distal to the SSR marker CAT_B501 on LG2 (Supplemental Fig. 2) (Sathuvalli et al., 2011 b). Additional work will be required to determine if these resistance genes are identical or allelic at the same locus, if they differ and are part of a cluster of disease resistance genes in that region, or if they differ in some other means from one another. Interestingly, the phenotypic response of OSU 759.010 in New Jersey differs from H3R07P25. In Molnar et al. (2010a), replications of an identical clone (OSU 759.007) expressed EFB after inoculation with most isolates in the study. In the field, Capik and Molnar (2012) also showed that it developed EFB; however, only four of six trees of OSU 759.007 developed EFB and their average proportion of diseased wood after 10 years of exposure was less than $1 \%$ of the tree canopy, which would qualify as resistant in this current study. Additional work with OSU 759.007 is currently under way to evaluate the similarity of these two resistance sources.

The findings from the current research, in combination with those from previous studies, have numerous important implications for european hazelnut breeders and growers. First, previous studies have shown differential EFB disease responses in the field between the 'Gasaway' resistance source and H3R07P25, different LG locations of resistance sources (LG6 vs. LG2, respectively), various pathogenic responses from different $A$. anomala isolates (Molnar et al., 2010a), and a high degree of fungal genetic diversity in EFB high disease pressure environments (Cai et al., 2013; Muehlbauer et al., 2014b, 2019; Tobia et al., 2017). These observations indicate that different isolates of $A$. anomala in high disease pressure environments may be eliciting differential pathogenic responses from european hazelnut plant material carrying different disease resistance genes. Although this was hypothesized in previous research (Capik and Molnar, 2012; Leadbetter et al., 2016; Molnar et al., 2010a, 2010b; Muehlbauer et al., 2014a; Sathuvalli et al., 2011a, 2011b), the current work further emphasizes the need for breeders to develop european hazelnut germplasm exhibiting EFB resistance via $R$-gene pyramiding approaches or through the identification of $R$-genes conferring resistance to multiple isolates of $A$. anomala (as indicated in the current work). Second, screening european hazelnut germplasm and developing new cultivars exhibiting EFB resistance in a high disease pressure environment with greater fungal genetic diversity may allow for the identification of germplasm capable of maintaining resistance against a greater number of fungal isolates. Third, the resistance conferred by the 'Gasaway' source is likely different from that identified in H3R07P25, does not appear to confer complete resistance to EFB in all high disease pressure environments, and therefore may confer resistance to only a limited number of $A$. anomala isolates. Accordingly, continuing efforts are needed to prevent the introduction of additional isolates of $A$. anomala into the 
PNW. In addition, should that effort fail, european hazelnut breeders need to continue to search for and identify new sources of EFB resistance that offer broader protection against genetically diverse EFB fungal populations. Fourth, identification of european hazelnut germplasm that maintains EFB resistance in high disease pressure environments is a requirement for the development of a commercially viable european hazelnut industry in the eastern United States. Identification of the LG location of that resistance, and transmission to progeny as a single locus with a dominant allele for resistance, will likely expedite that development. Last, the identification of genetic markers tightly linked to EFB resistance, as well as previously discovered linked genetic markers, will help facilitate resistance gene pyramiding approaches (e.g., 'Gasaway'-based resistance from LG6, 'Rotoli'-based resistance from LG7, and H3R07P25-based resistance from LG2 in the current work) via marker-aided breeding, and/or the identification of EFB resistance genes that confer resistance to multiple $A$. anomala isolates.

\section{Literature Cited}

Akin, M., A. Nyberg, J. Postman, S. Mehlenbacher, and N.V. Bassil. 2016. A multiplexed microsatellite fingerprinting set for hazelnut cultivar identification. Eur. J. Hort. Sci. 81:327-338.

Bassil, N., P. Boccacci, R. Botta, J. Postman, and S. Mehlenbacher. 2013. Nuclear and chloroplast microsatellite markers to assess genetic diversity and evolution in hazelnut species, hybrids and cultivars. Genet. Resources Crop Evol. 60:543-568.

Bassil, N.V., R. Botta, and S.A. Mehlenbacher. 2005. Microsatellite markers in hazelnut: Isolation, characterization and cross-species amplification. J. Amer. Soc. Hort. Sci. 130:543-549.

Beltramo, C., N. Valentini, E. Portis, D. Torello Marinoni, P. Boccacci, M.A. Sandoval Prando, and R. Botta. 2016. Genetic mapping and QTL analysis in european hazelnut (Corylus avellana L.). Mol. Breed. 36:27.

Bhattarai, G. 2015. Microsatellite marker development, characterization and mapping in european hazelnut (Corylus avellana L.), and investigation of novel sources of eastern filbert blight resistance in Corylus. Oregon State Univ., Corvallis, MS Thesis.

Bhattarai, G. and S.A. Mehlenbacher. 2017. In silico development and characterization of tri-nucleotide simple sequence repeat markers in hazelnut (Corylus avellana L.). PLoS One 12:e178061.

Bhattarai, G. and S.A. Mehlenbacher. 2018. Discovery, characterization and linkage mapping of simple sequence repeat markers in hazelnut. J. Amer. Soc. Hort. Sci. 143:347-362.

Bhattarai, G., S.A. Mehlenbacher, and D.C. Smith. 2017. Eastern filbert blight disease resistance from Corylus americana 'Rush' and selection 'Yoder \#5' maps to linkage group 7. Tree Genet. Genomes $13: 45$.

Boccacci, P., A. Akkak, N.V. Bassil, S.A. Mehlenbacher, and R. Botta. 2005. Characterization and evaluation of microsatellite loci in european hazelnut (Corylus avellana L.) and their transferability to other Corylus species. Mol. Ecol. Notes 5:934-937.

Brownstein, M.J., J.D. Carpten, and J.R. Smith. 1996. Modulations of non-templated nucleotide addition by Taq DNA polymerase: Primer modifications that facilitate genotyping. Biotechniques 20:10041010.

Cai, G., C.W. Leadbetter, M.F. Muehlbauer, T.J. Molnar, and B.I. Hillman. 2013. Genome-wide microsatellite identification in the fungus Anisogramma anomala using Illumina sequencing and genome assembly. PLoS One 8(11):e82408, doi: 10.1371/journal. pone. 0082408 .

Capik, J.M. and T.J. Molnar. 2012. Assessment of host (Corylus sp.) resistance to eastern filbert blight in New Jersey. J. Amer. Soc. Hort. Sci. 137:157-172.
Capik, J.M. and T.J. Molnar. 2014. Flowering phenology of eastern filbert blight-resistant hazelnut accessions in New Jersey. HortTechnology 24:196-208.

Capik, J.M., M. Muehlbauer, A. Novy, J.A. Honig, and T.J. Molnar. 2013. Eastern filbert blight-resistant hazelnuts from Russia, Ukraine, and Poland. HortScience 48:466-473.

Cameron, H.R. 1976. Eastern filbert blight established in the Pacific northwest. Plant Dis. Rptr. 60:737-740.

Catchen, J., A. Amores, P. Hohenlohe, W. Cresko, and J. Postlethwait. 2011. Stacks: Building and genotyping loci de novo from short-read sequences. Genes Genomes Genet. 1:171-182.

Catchen, J., P.A. Hohenlohe, S. Bassham, A. Amores, and W.A. Cresko. 2013. Stacks: An analysis toolset for population genomics. Mol. Ecol. 22:3124-3140.

Chen, H., S.A. Mehlenbacher, and D.C. Smith. 2007. Hazelnut accessions provide new sources of resistance to eastern filbert blight. HortScience 42:466-469.

Chen, Z., S.R. Manchester, and H. Sun. 1999. Phylogeny and evolution of the Betulaceae as inferred from DNA sequences, morphology, and paleobotany. Amer. J. Bot. 86:1168-1181.

Ciarmiello, L.F., M.F. Mazzeo, P. Minasi, A. Peluso, A. De Luca, P. Piccirillo, R.A. Siciliano, and V. Carbone. 2014. Analysis of different european hazelnut (Corylus avellana L.) cultivars: Authentication, phenotypic features, and phenolic profiles. J. Agr. Food Chem. 62:6236-6246.

Colburn, B.C., S.A. Mehlenbacher, and V.R. Sathuvalli. 2017. Development and mapping of microsatellite markers from transcriptome sequences of european hazelnut (Corylus avellana L.) and use for germplasm characterization. Mol. Breed. 37:16-29.

Colburn, B.C., S.A. Mehlenbacher, V.R. Sathuvalli, and D.C. Smith. 2015. Eastern filbert blight resistance in hazelnut accessions 'Culplà', 'Crvenje' and OSU 495.072. J. Amer. Soc. Hort. Sci. 140:191-200.

Coyne, C.J., S.A. Mehlenbacher, and D.C. Smith. 1998. Sources of resistance to eastern filbert blight. J. Amer. Soc. Hort. Sci. 124:253257.

Davison, A.D. and R.M. Davidson. 1973. Apioporthe and Monchaetia canker reported in western Washington. Plant Dis. Rptr. 57:522-523.

Elshire, R.J., J.C. Glaubitz, Q. Sun, J.A. Poland, K. Kawamoto, E.S. Buckler, and S.E. Mitchell. 2011. A robust, simple genotyping-bysequencing (GBS) approach for high diversity species. PLoS One 6(5):e19379, doi: 10.1371/journal.pone.0019379.

Erdogan, V. and S.A. Mehlenbacher. 2000. Phylogenetic relationships of Corylus species (Betulaceae) based on nuclear ribosomal DNA ITS region and chloroplast matK gene sequences. Syst. Bot. 25:727737.

Food and Agricultural Organization of the United Nations. 2016. FAOSTAT. 7 Dec. 2018. <http://www.fao.org/faostat/en/?data/QC>. Fuller, A.S. 1908. The nut culturist. Orange Judd, New York, NY.

Gottwald, T.R. and H.R. Cameron. 1980. Disease increase and the dynamics of spread of canker caused by Anisogramma anomala in european filbert in the Pacific northwest. Phytopathology 70:10871092 .

Grattapaglia, D. and R. Sederoff. 1994. Genetic linkage maps of Eucalyptus grandis and Eucalyptus urophylla using a pseudotestcross: Mapping strategy and RAPD markers. Genetics 137:1121-1137.

Gürcan, K. and S.A. Mehlenbacher. 2010. Development of microsatellite marker loci for european hazelnut (Corylus avellana L.) from ISSR fragments. Mol. Breed. 26:551-559.

Gürcan, K., S.A. Mehlenbacher, R. Botta, and P. Boccacci. 2010a. Development, characterization, segregation, and mapping of microsatellite markers for european hazelnut (Corylus avellana L.) from enriched genomic libraries and usefulness in genetic diversity studies. Tree Genet. Genomes 6:513-531.

Gürcan, K., S.A. Mehlenbacher, and V. Erdogan. 2010b. Genetic diversity in hazelnut (Corylus avellana L.) cultivars from Black Sea countries assessed using SSR markers. Plant Breed. 129:422-434. 
Halsted, B.D. 1892. A serious filbert disease. New Jersey Agr. Expt. Sta. Annu. Rpt. 13:287-288.

Herrmann, D., B. Boller, B. Studer, F. Widmer, and R. Kolliker. 2006. QTL analysis of seed yield components in red clover (Trifolium pratense L.). Theor. Appl. Genet. 112:536-545.

Ives, C., V.R. Sathuvalli, B.C. Colburn, and S.A. Mehlenbacher. 2014. Mapping the incompatibility and style color loci in two hazelnut progenies. HortScience 49:250-253.

Johnson, K.B., S.A. Mehlenbacher, J.K. Stone, and J.W. Pscheidt. 1996. Eastern filbert blight of european hazelnut: It's becoming a manageable disease. Plant Dis. 80:1308-1316.

Johnson, K.B. and J.N. Pinkerton. 2002. Eastern filbert blight, p. 44 46. In: B.L. Teviotdale, T.J. Michailides, and J.W. Pscheidt (eds.). Compendium of nut crop diseases in temperate zone. APS Press, St. Paul, MN.

Julian, J.W., C.F. Seavert, and J.L. Olsen. 2008. Orchard economics: The costs and returns of establishing and producing hazelnuts in the Willamette Valley. Oregon State Univ. Ext. Bul. EM 8748-E.

Leadbetter, C.W., J.M. Capik, S.A. Mehlenbacher, and T.J. Molnar. 2016. Hazelnut accessions from Russia and Crimea transmit resistance to eastern filbert blight. J. Am. Pomol. Soc. 70:92-109.

Leinonen, R., H. Sugawara, M. Shumway, (on behalf of the International Nucleotide Sequence Database Collaboration). 2011. The sequence read archive. Nucleic Acids Res. 39:D19-D20.

Lunde, C.F., S.A. Mehlenbacher, and D.C. Smith. 2000. Survey of hazelnut cultivars for response to eastern filbert blight inoculation. HortScience 35:729-731.

Lunde, C.F., S.A. Mehlenbacher, and D.C. Smith. 2006. Segregation for resistance to eastern filbert blight in progeny of 'Zimmerman' hazelnut. J. Amer. Soc. Hort. Sci. 131:731-737.

Margarido, G.R.A., A.P. Souza, and A.A.F. Garcia. 2007. OneMap: Software for genetic mapping in outcrossing species. Hereditas 144:78-79.

McAdam, E.L., J.S. Freeman, S.P. Whittock, E.J. Buck, J. Jakse, A. Cerenak, B. Javornik, A. Kilian, C.-H. Wang, D. Andersen, R.E. Vaillancourt, J. Carling, R. Beatson, L. Graham, D. Graham, P. Darby, and A. Koutoulis. 2013. Quantitative trait loci in hop (Humulus lupulus L.) reveal complex genetic architecture underlying variation in sex, yield and cone chemistry. BMC Genomics 14:360.

Mehlenbacher, S.A. 1991. Hazelnuts (Corylus), p. 789-836. In: J.N. Moore and J.R. Ballington (eds.). Genetic resources of temperate fruit and nut crops. Intl. Soc. Hort. Sci., Wageningen, The Netherlands.

Mehlenbacher, S.A. 1994. Genetic improvement of the hazelnut. Acta Hort. 351:23-38.

Mehlenbacher, S.A. 1995. Progress in breeding new hazelnut cultivars in Oregon. Nucis Newsl. 3:8-9.

Mehlenbacher, S.A., A.N. Azarenko, D.C. Smith, and R. McCluskey. 2000. 'Lewis' hazelnut. HortScience 35:314-315.

Mehlenbacher, S.A., R.N. Brown, E.R. Nouhra, T. Gökirmak, N.V. Bassil, and T.L. Kubisiak. 2006. A genetic linkage map for hazelnut (Corylus avellana L.) based on RAPD and SSR markers. Genome 49:122-133.

Mehlenbacher, S.A. and D.C. Smith. 2004. Hazelnut pollenizers 'Gamma', 'Delta', 'Epsilon', and 'Zeta'. HortScience 39:14981499.

Mehlenbacher, S.A., D.C. Smith, and R.L. McCluskey. 2008. 'Sacajawea' hazelnut. HortScience 43:255-257.

Mehlenbacher, S.A., D.C. Smith, and R.L. McCluskey. 2009. 'Yamhill' hazelnut. HortScience 44:845-847.

Mehlenbacher, S.A., D.C. Smith, and R.L. McCluskey. 2011. 'Jefferson' hazelnut. HortScience 46:662-664.

Mehlenbacher, S.A., D.C. Smith, and R.L. McCluskey. 2012. 'Eta' and 'Theta' hazelnut pollenizers. HortScience 47:1180-1181.

Mehlenbacher, S.A., D.C. Smith, and R.L. McCluskey. 2013. 'Dorris' hazelnut. HortScience 48:796-799.

Mehlenbacher, S.A., D.C. Smith, and R.L. McCluskey. 2014. 'Wepster' hazelnut. HortScience 49:346-349.
Mehlenbacher, S.A., D.C. Smith, and R.L. McCluskey. 2016. 'McDonald' hazelnut. HortScience 51:757-760.

Mehlenbacher, S.A. and M.M. Thompson. 1991. Four hazelnut pollenizers resistant to eastern filbert blight. HortScience 26:442443.

Mehlenbacher, S.A., M.M. Thompson, and H.R. Cameron. 1991. Occurrence and inheritance of resistance to eastern filbert blight in 'Gasaway' hazelnut. HortScience 26:410-411.

Molnar, T.J. and J.M. Capik. 2012. Eastern filbert blight susceptibility of american $\times$ european hazelnut progenies. HortScience 47:14121418.

Molnar, T.J., J.M. Capik, S. Zhao, and N. Zhang. 2010b. First report of eastern filbert blight on Corylus avellana 'Gasaway' and 'VR2011' caused by Anisogramma anomala in New Jersey. Plant Dis. 10:1265.

Molnar, T.J., J.C. Goffreda, and C.R. Funk. 2010a. Survey of Corylus resistance to Anisogramma anomala from different geographic regions. HortScience 45:832-836.

Molnar, T.J., S.A. Mehlenbacher, D.E. Zaurov, and J.C. Goffreda. 2007. Survey of hazelnut germplasm from Russia and Crimea for response to eastern filbert blight. HortScience 42:51-56.

Molnar, T.J., E. Walsh, J.M. Capik, V. Sathuvalli, S.A. Mehlenbacher, A.Y. Rossman, and N. Zhang. 2013. A real-time PCR assay for early detection of eastern filbert blight. Plant Dis. 97:813-818.

Molnar, T.J., J.J. Lombardoni, M.F. Muehlbauer, J.A. Honig, S.A. Mehlenbacher, and J.M. Capik. 2018. Progress breeding for resistance to eastern filbert blight in the eastern United States. Acta Hort. 1226:79-85.

Muehlbauer, M., J.M. Capik, T.J. Molnar, and S.A. Mehlenbacher. 2018. Assessment of the 'Gasaway' source of resistance to eastern filbert blight in New Jersey. Scientia Hort. 235:367-372.

Muehlbauer, M.F., J.A. Honig, J.M. Capik, J.N. Vaiciunas, and T.J. Molnar. 2014a. Characterization of eastern filbert blight-resistant hazelnut germplasm using microsatellite markers. J. Amer. Soc. Hort. Sci. 139:399-432.

Muehlbauer, M., T.J. Molnar, J. Honig, K. Morey, and N. Zhang. 2014b. Genetic diversity of Anisogramma anomala and its implications for breeding eastern filbert blight resistant hazelnuts. 2014. HortScience 49:S134, (Abstr.).

Muehlbauer, M.F., J. Tobia, J.A. Honig, N. Zhang, B.I. Hillman, K. Morey Gold, and T.J. Molnar. 2019. Population differentiation within Anisogramma anomala in North America. Phytopathology 109:1074-1082.

Osterbauer, N.K. 1996. Genetic variability in the eastern filbert blight pathosystem. PhD Diss., Oregon State Univ., Corvallis.

Osterbauer, N.K., K.B. Johnson, S.A. Mehlenbacher, and T.L. Sawyer. 1997. Analysis of resistance to eastern filbert blight in Corylus avellana. Plant Dis. 81:388-394.

Petriccione, M., L.F. Ciarmiello, P. Boccacci, A. De Luca, and P. Piccirillo. 2010. Evaluation of 'Tonda di Giffoni' hazelnut (Corylus avellana L.) clones. Scientia Hort. 124:153-158.

Pinkerton, J.N., K.B. Johnson, K.M. Theiling, and J.A. Griesbach. 1992. Distribution and characteristics of the eastern filbert blight epidemic in western Oregon. Plant Dis. 76:1179-1182.

Pinkerton, J.N., K.B. Johnson, S.A. Mehlenbacher, and J.W. Pscheidt. 1993. Susceptibility of european hazelnut clones to eastern filbert blight. Plant Dis. 77:261-266.

Pinkerton, J.N., K.B. Johnson, J.K. Stone, and K.L. Ivors. 1998. Maturation and seasonal discharge pattern of ascospores of Anisogramma anomala. Phytopathology 88:1165-1173.

Poland, J.A., P.J. Brown, M.E. Sorrells, and J.-L. Jannink. 2012. Development of high-density genetic maps for barley and wheat using a novel two-enzyme genotyping-by-sequencing approach. PLoS One 7(2):e32253, doi: 10.1371/journal.pone.0032253.

Rowley, E.R., R. VanBuren, D.W. Bryant, H.D. Priest, S.A. Mehlenbacher, and T.C. Mockler. 2018. A draft genome and high-density genetic map of european hazelnut (Corylus avellana L.). BioRxiv 469015 (preprint). 11 Dec. 2018 (cited 21 Dec. 2018). 
Sathuvalli, V.R. and S.A. Mehlenbacher. 2013. De novo sequencing of hazelnut bacterial artificial chromosomes (BACs) using multiplex Illumina sequencing and targeted marker development for eastern filbert blight resistance. Tree Genet. Genomes 9:1109-1118.

Sathuvalli, V.R., H. Chen, S.A. Mehlenbacher, and D.C. Smith. 2011a. DNA markers linked to eastern filbert blight resistance in 'Ratoli' hazelnut (Corylus avellana L.). Tree Genet. Genomes 7:337-345.

Sathuvalli, V.R., S.A. Mehlenbacher, and D.C. Smith. 2011b. DNA markers linked to eastern filbert blight resistance from a hazelnut selection from the Republic of Georgia. J. Amer. Soc. Hort. Sci. 136:350-357.

Sathuvalli, V.R., S.A. Mehlenbacher, and D.C. Smith. 2010. Response of hazelnut accessions to greenhouse inoculation with Anisogramma anomala. HortScience 45:1116-1119.

Sathuvalli, V.R., S.A. Mehlenbacher, and D.C. Smith. 2012. Identification and mapping of DNA markers linked to eastern filbert blight resistance from OSU 408.040 hazelnut. HortScience 47:570-573.

Sathuvalli, V.R., S.A. Mehlenbacher, and D.C. Smith. 2017. Highresolution genetic and physical mapping of the eastern filbert blight resistance region in 'Jefferson' hazelnut (Corylus avellana L.). Plant Genome 10(2):doi: 10.3835/plantgenome2016.12.0123.

Schuelke, M. 2000. An economic method for the fluorescent labeling of PCR fragments. Nat. Biotechnol. 18:233-234.

Studer, B., B. Boller, D. Herrmann, E. Bauer, U.K. Posselt, F. Widmer, and R. Kolliker. 2006. Genetic mapping reveals a single major QTL for bacterial wilt resistance in Italian ryegrass (Lolium multiflorum Lam.). Theor. Appl. Genet. 113:661-671.

Thompson, M.M., H.B. Lagerstedt, and S.A. Mehlenbacher. 1996. Hazelnuts, p. 125-184. In: J. Janick and J.N. Moore (eds.). Fruit breeding. Vol. 3. Nuts. Wiley, New York, NY.

Tobia, J., M. Muehlbauer, J. Honig, J. Pscheidt, and T.J. Molnar. 2017. Cluster analysis of Anisogramma anomala isolates collected from the Pacific northwest and New Jersey. Phytopathology 107:S5.125 (Abstr.).
Torello Marinoni, D., N. Valentini, E. Portis, A. Acquadro, C. Beltramo, S.A. Mehlenbacher, T.C. Mockler, E.R. Rowley, and R. Botta. 2018. High density SNP mapping and QTL analysis for time of leaf budburst in Corylus avellana L. PLoS One 13(4):e0195408, doi: 10.1371/journal.pone.0195408.

U.S. Department of Agriculture. 2018. Noncitrus fruits and nuts 2017 summary. Natl. Agr. Stat. Serv., U.S. Dept. Agr., Washington, DC. van Heerden, C.J., P. Burger, A. Vermeulen, and R. Prins. 2014. Detection of downy and powdery mildew resistance QTL in a 'Regent' x 'RedGlobe' population. Euphytica 200:281-295.

van Ooijen, J.W. 2006. JoinMap 4, Software for the calculation of genetic linkage maps in experimental populations. Kyazma, Wageningen, The Netherlands.

van Ooijen, J.W. 2009. MapQTL 6, Software for the mapping of quantitative trait loci in experimental populations of diploid species. Kyazma, Wageningen, The Netherlands.

van Ooijen, J.W. 2011a. Multipoint maximum likelihood mapping in a full-sib family of an outbreeding species. Genet. Res. 93:343-349.

van Ooijen, J.W. 2011b. JoinMap 4.1: New features. 28 Sept. 2018. $<$ https://www.kyazma.nl/docs/JM41Features.pdf $>$.

Voorips, R.E. 2002. MapChart: Software for the graphical presentation of linkage maps and QTLs. J. Hered. 93:77-78.

Yoo, K. and J. Wen. 2002. Phylogeny and biogeography of Carpinus and subfamily Coryloideae (Betulaceae). Intl. J. Plant Sci. 163:641650.

Yun, L., S.R. Larson, I.W. Mott, K.B. Jensen, and J.E. Staub. 2014. Genetic control of rhizomes and genomic localization of a majoreffect growth habit QTL in perennial wildrye. Mol. Genet. Genomics 289:383-397.

Zyprian, E., I. Ochbner, F. Schwander, S. Simon, L. Hausmann, M. Bonow-Rex, P. Moreno-Sanz, M. Stella Grando, S. WiedemannMerdinoglu, D. Merdinoglu, R. Eibach, and R. Topfer. 2016. Quantitative trait loci affecting pathogen resistance and ripening of grapevines. Mol. Genet. Genomics 291:1573-1594. 

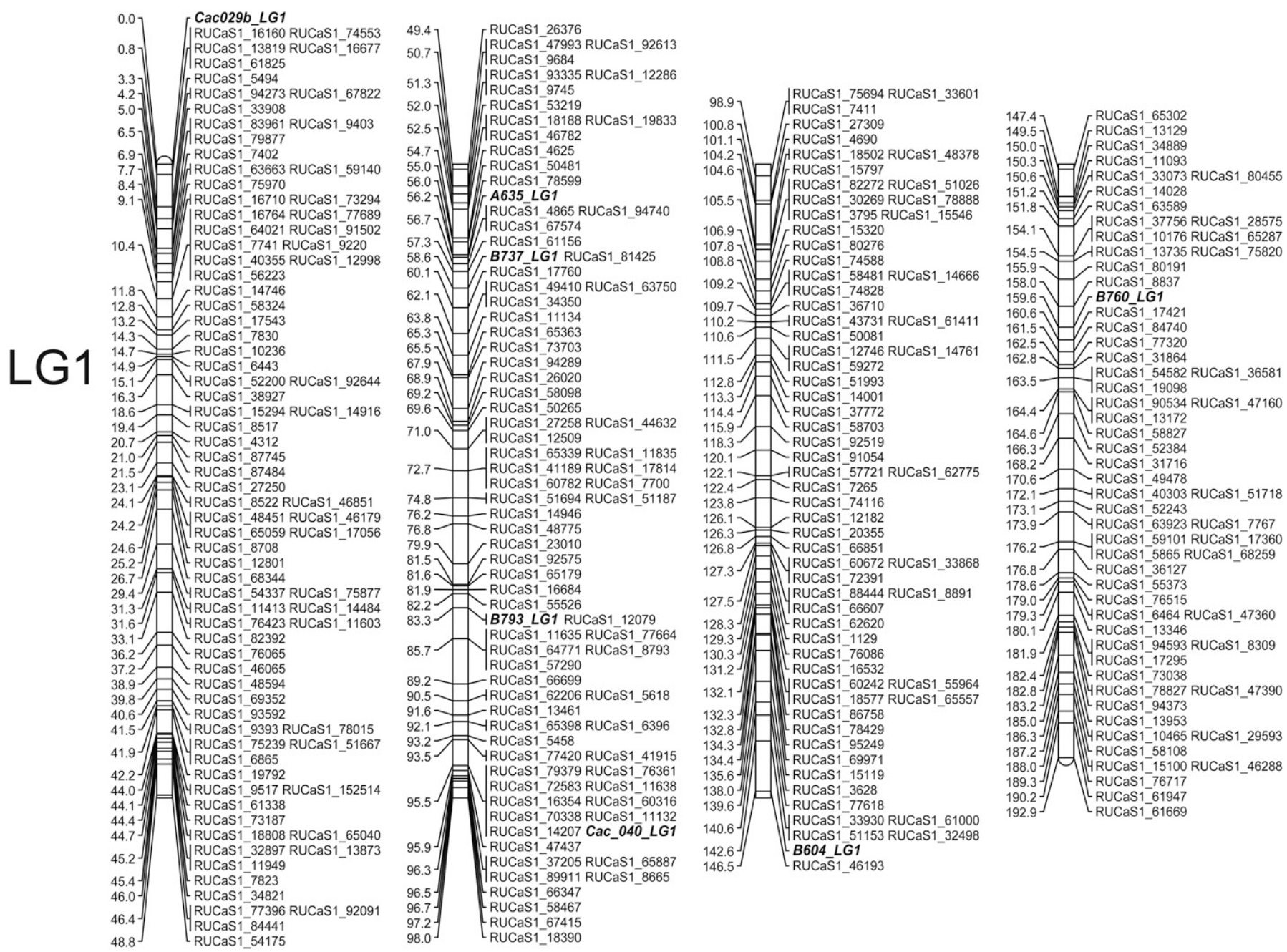
154.5_LC13735 RUCaS1_75820 155.9 RUCaS1_80191 158.0 RUCaS1 8837 160.6] RUCaS1 17421 161.5] RUCaS1 184740 162.5 RUCaS1 177320 162.8 RUCaS1 31864 163.5 RUCaS1_54582 RUCaS1_36581 164.4 RRUCaS1_90534 RUCaS1_47160 RUCaS1 13172 164.6 RUCaS1 58827 166.3 RUCaS1 52384 168.2 RUCaS1 31716 - RUCAS1_40303 RUCaS1 51718 173.1 RUCaS1 52243

173.9 YRUCaS1_63923 RUCaS1_7767 176.2 RUCaS1_59101 RUCaS1_17360 176.8 ${ }_{\text {RUCaS1 } 36127}$ 178.6 A RUCaS1_55373 179.0 _RUCaS1_76515 179.3 $]$ 目 $[4 \mathrm{RUCaS1} 6464$ RUCaS1_47360 180.1 1 [ RUCaS1_13346 182.4 $]$ RUCaS1_17295

182.8 E-4RUCaS1_78827 RUCaS1_47390 183.2 B RUCaS1_94373 185.0 $\rightarrow$ RUCaS1_13953

186.3 IRUCaS1_10465 RUCaS1_29593 187.2 LRUCaS1 58108 188.0 4RUCas1_15100 RUCaS1_46288 189.3 RUCaS1_76717

190.2] RUCaS1_61947 192.9 [RUCaS1_61669

Supplemental Fig. 1. Representation of european hazelnut linkage group (LG) 1. Microsatellite or simple sequence repeat (SSR) anchor markers are highlighted in bold italics. 

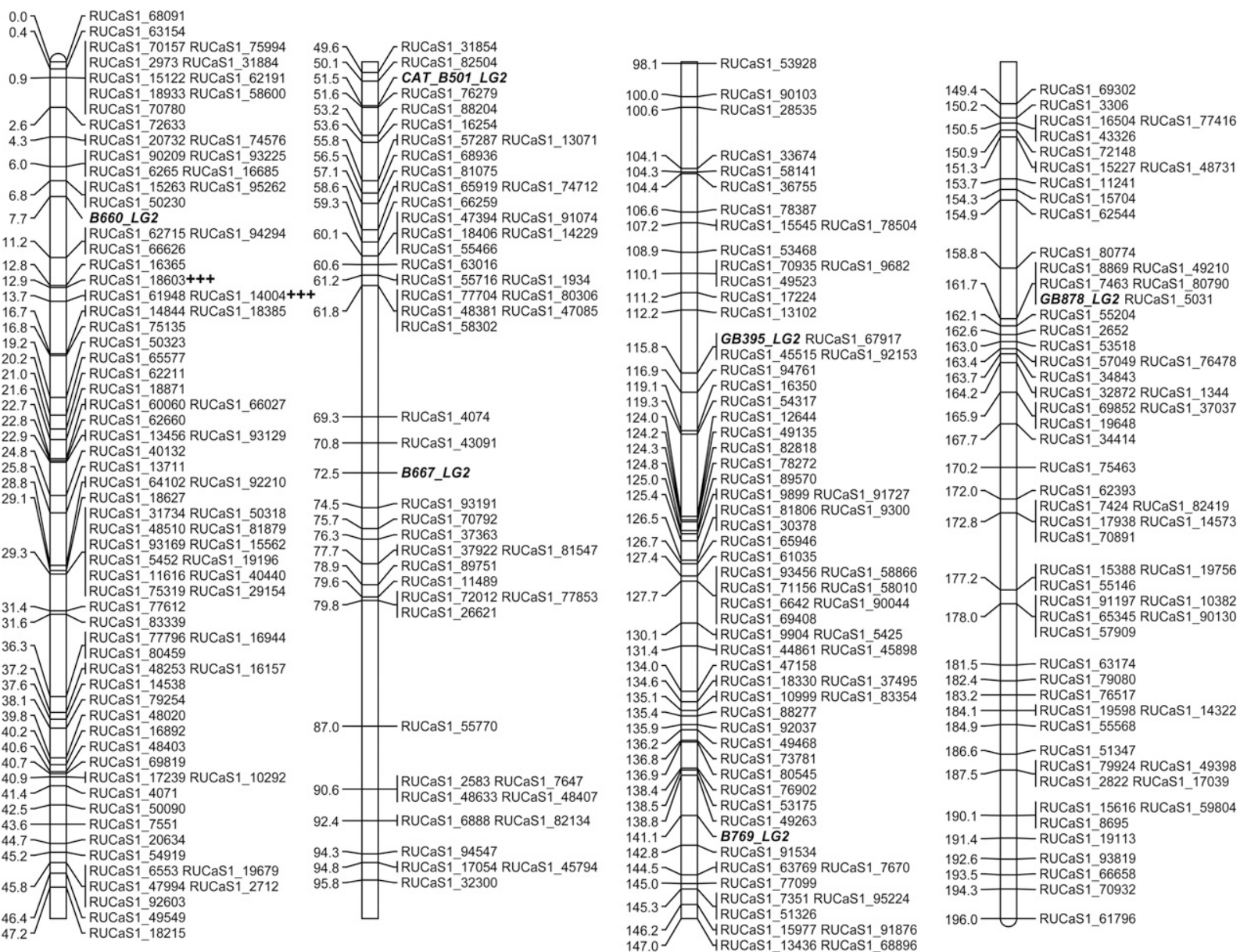

Supplemental Fig. 2. Representation of european hazelnut linkage group (LG) 2. The eastern filbert blight (EFB) disease resistance quantitative trait loci (QTL) region is denoted by "+++." Microsatellite or simple sequence repeat (SSR) anchor markers are highlighted in bold italics. 


\section{LG3 [1]}

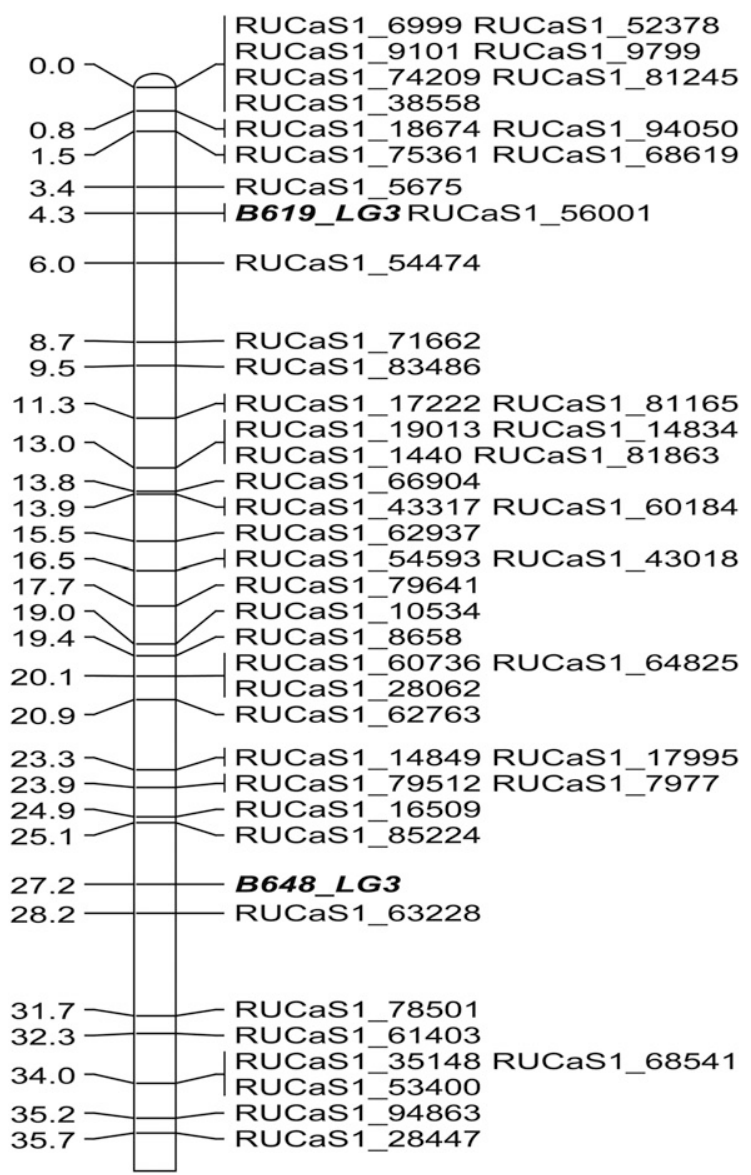

\section{LG3 [2]}

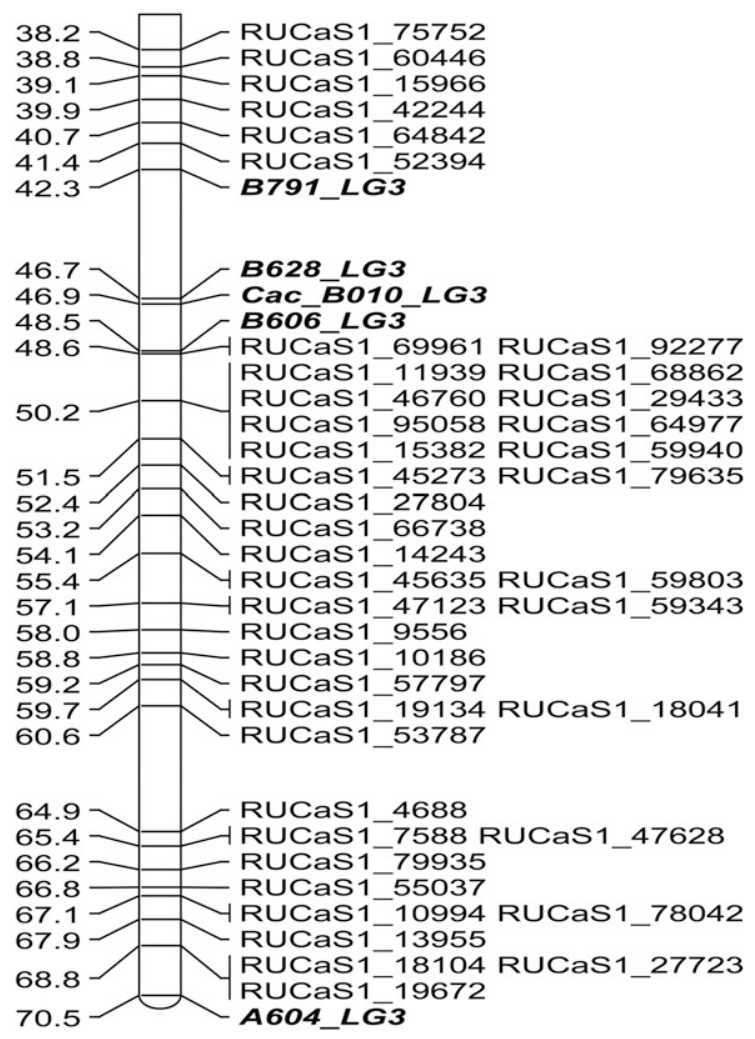

Supplemental Fig. 3. Representation of european hazelnut linkage group (LG) 3. Microsatellite or simple sequence repeat (SSR) anchor markers are highlighted in bold italics. 

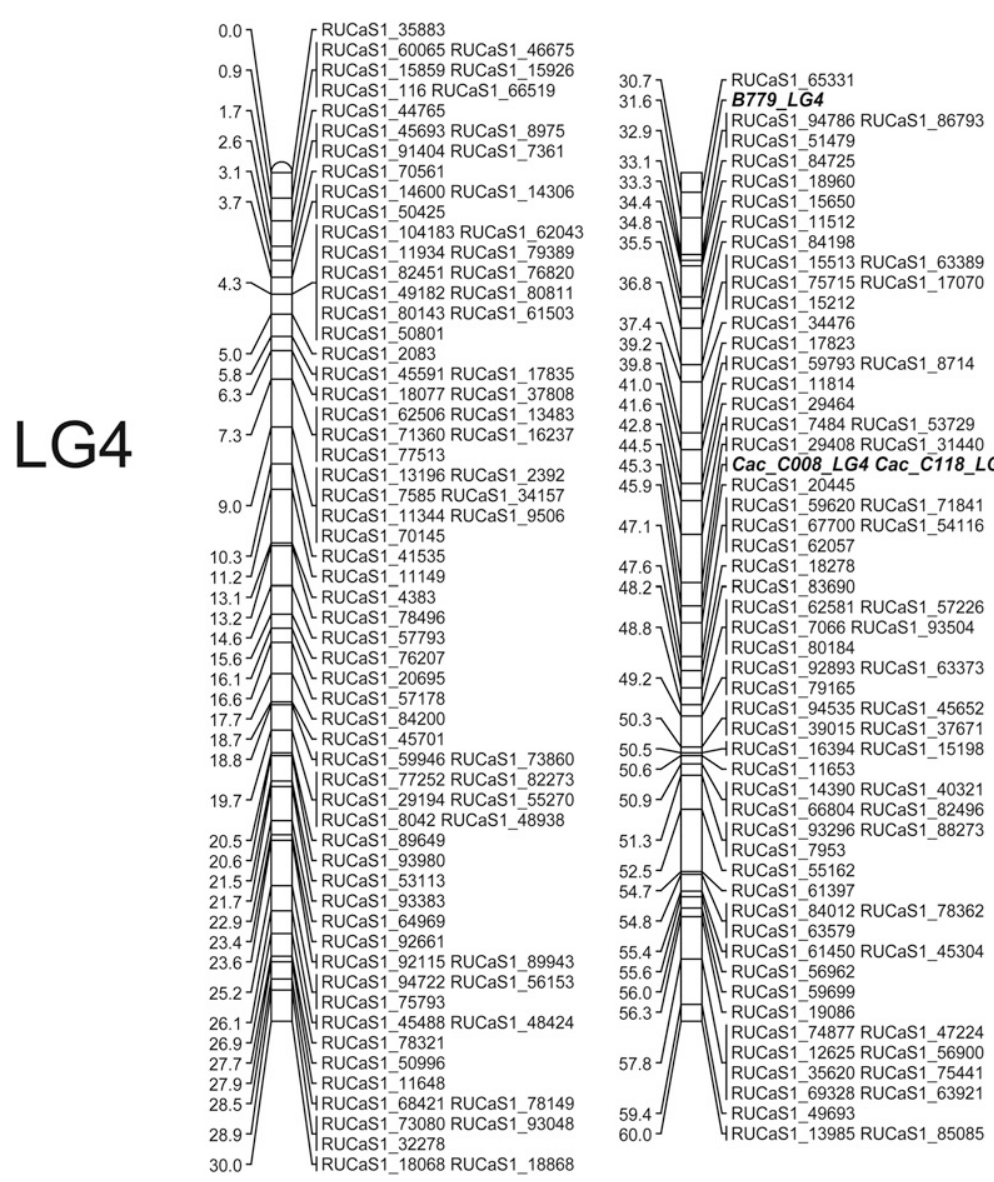
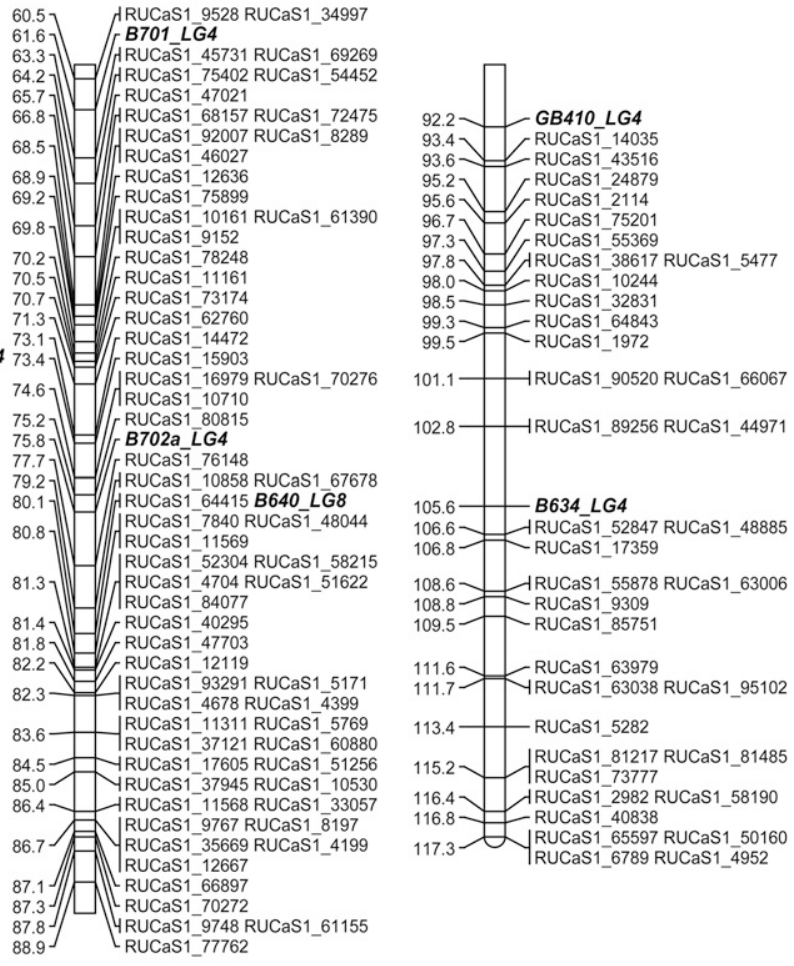
101.1 - - RUCaS1_90520 RUCaS1_66067 102.8-لIRUCaS1_89256 RUCaS1_44971

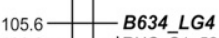
106.6 106.8 ${ }_{\text {RUCaS1-17359 }}$

108.6_-IRUCaS1_55878 RUCaS1_63006 108.8-RUCaS1_9309 109.5 - RUCaS1_85751

111.6 RUCaS1 63979 111.7 TRUCaS1_63038 RUCaS1_95102 113.4 - RUCaS1_5282 115.2 RUCaS1_81217 RUCaS1_81485 1164 RUCaS1_73777 RUCas1_81485 116.4 - IRUCaS1_2982 RUC 117.3 RUCaS1_65597 RUCaS1 50160 RUCaS1_6789 RUCaS1_4952

Supplemental Fig. 4. Representation of european hazelnut linkage group (LG) 4. Microsatellite or simple sequence repeat (SSR) anchor markers are highlighted in bold italics. 


\section{LG5 [1]}

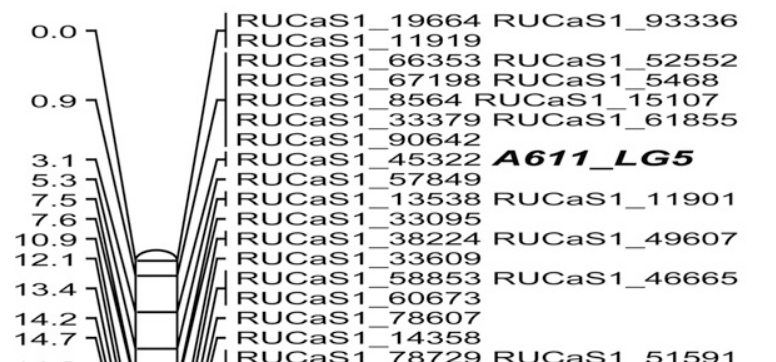
LG5 RUCaS1-78729 RUCaS1_51591 $14.8]-\left[\begin{array}{lll}R U C a S 1-38572 \\ \text { RuCas }\end{array}\right.$

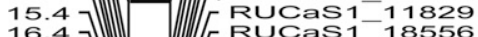

$\left.\begin{array}{l}16.4 \\ 16.7\end{array}\right]||-|||-|$ RuCas1-18556

17.5 $18.2] \mid$ RUCas1_46464 RUCas1_12351

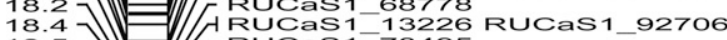
18.5 W $=$ RUCas1_78495

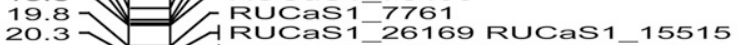
$21.3 \quad$ RuCas1_18085 RuCas1_58154 - RuCas1-13600 $21.7=$ RuCas1_63534

22.8 RUCaS1_82313 RUCas1_18182 23.9 MUCas1_48893 RUCas1_64441 $24.0] /$ RUCas1_42586

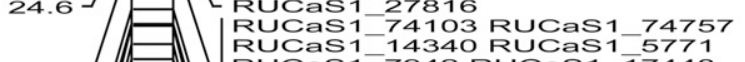

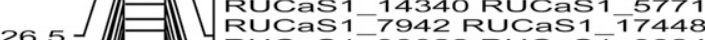
RUCaS1-38022 RUCaST 9824 RuCas1_9226 RuCas1_50492 RUCaS1-94679

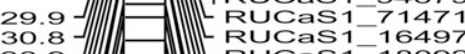
$30.8]$

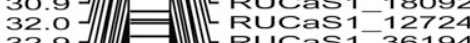

33.6 $D$ RUCas 1-12588 RUCas1 28478

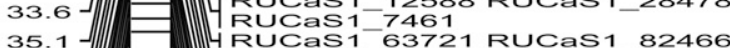
35. 1

37.3

38.1

39.5

41.5

41.7

42.5

44.2

45. 1

45.9

49.2 RUCas1_9265 RUCas1 71507 4 RUCaS1-9709 I RUCaS1_13813 RUCas1_59837 RUCas1-10553 H RUCaS1_80393 RUCaS1_75288 RUCaS1_30027 RUCas1-52750 I RUCas1- 45621 RUCas1-79728 RUCas1_69731 RUCasi_42828 BU4T1 LG5

\section{LG5 [2]}

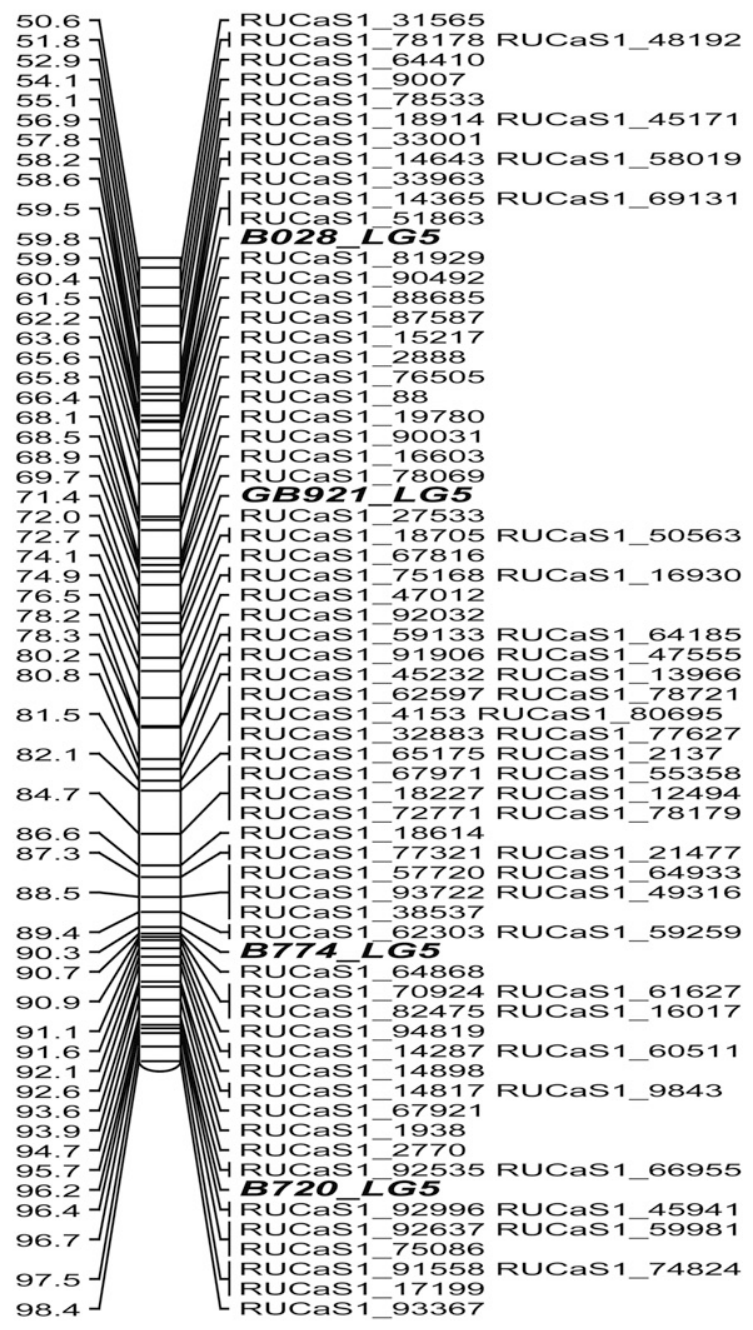

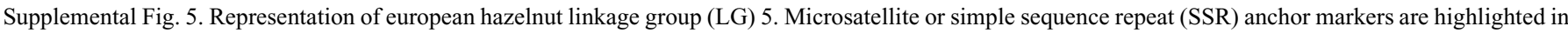
bold italics. 

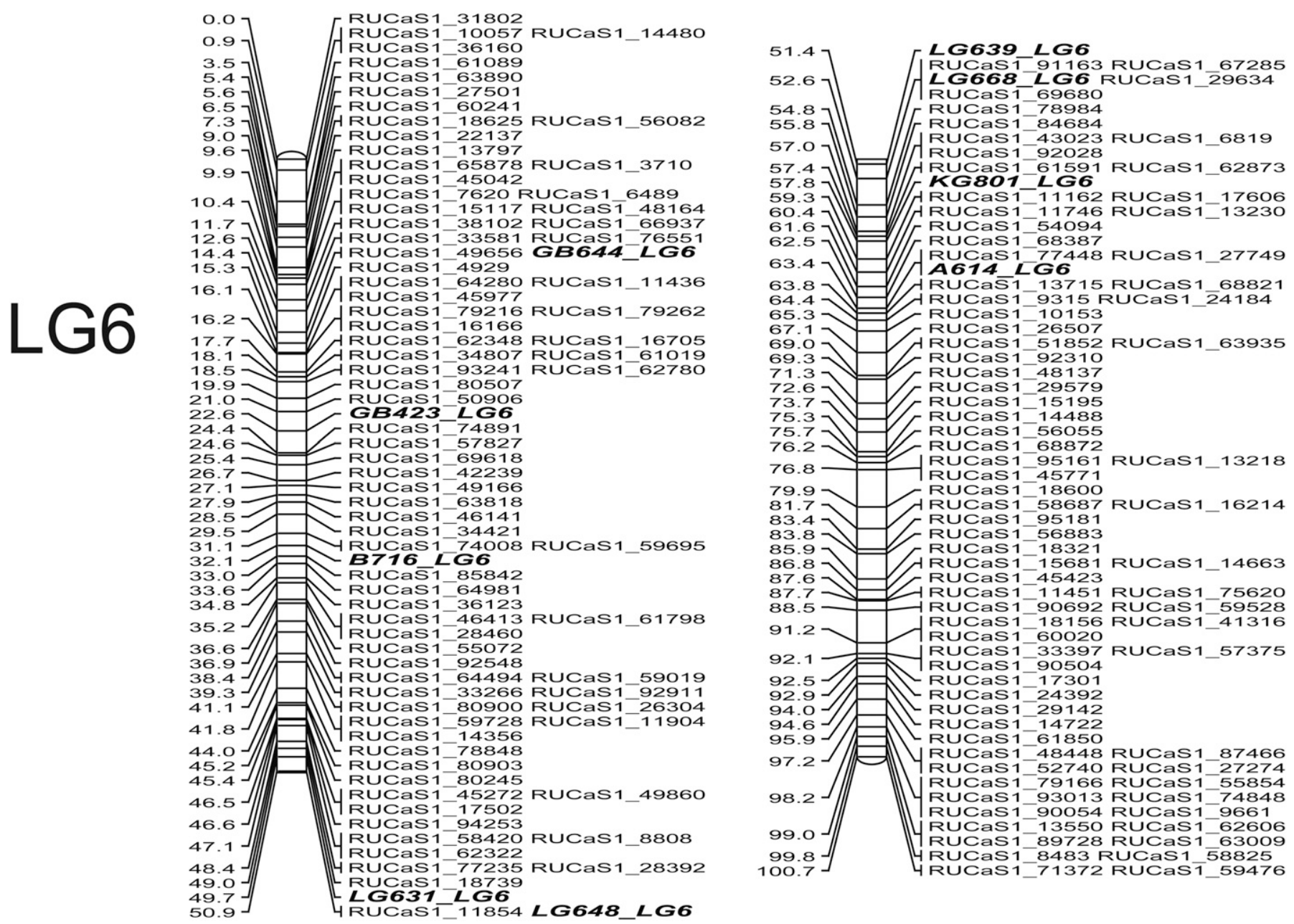

Supplemental Fig. 6. Representation of european hazelnut linkage group (LG) 6. Microsatellite or simple sequence repeat (SSR) anchor markers are highlighted in bold italics. 


\section{LG7 [1]}

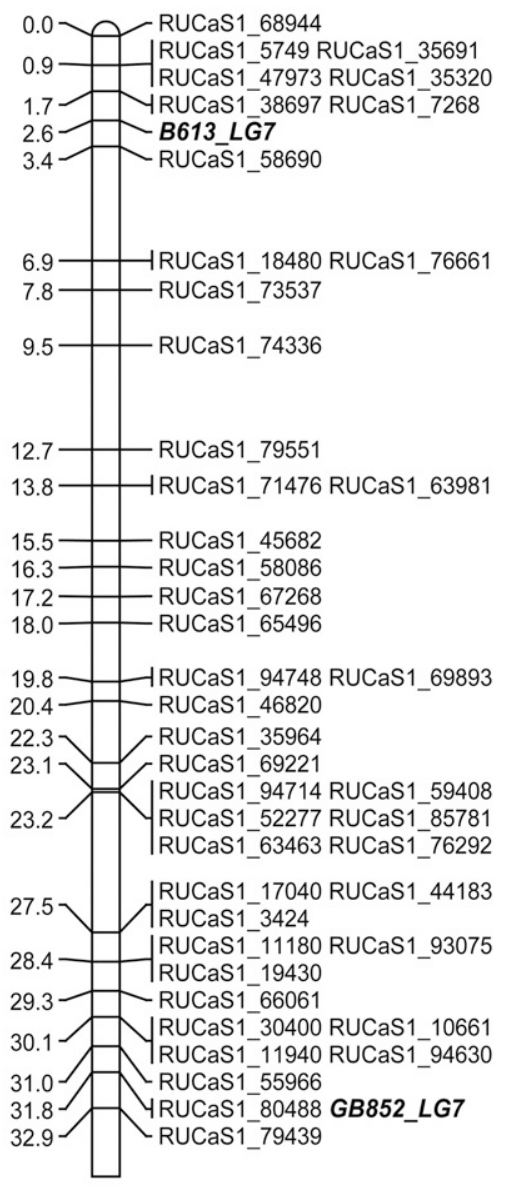

LG7 [2]

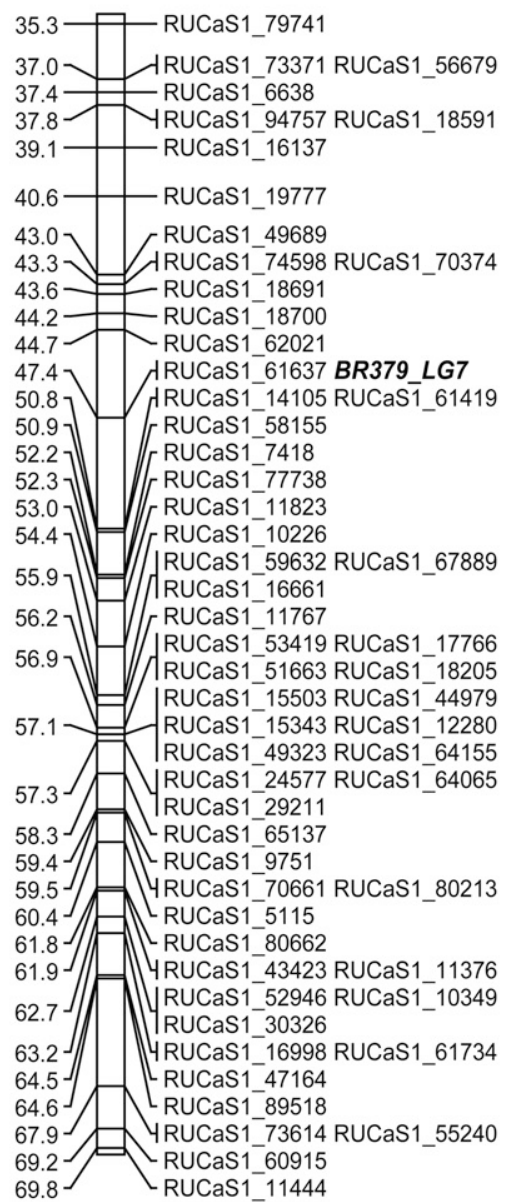

LG7 [3]

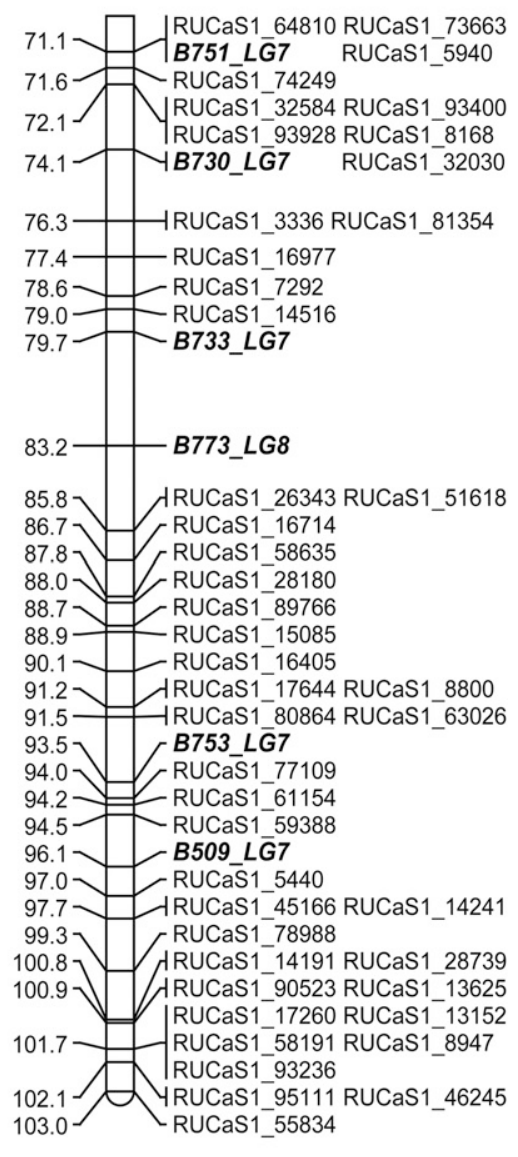

Supplemental Fig. 7. Representation of european hazelnut linkage group (LG) 7. Microsatellite or simple sequence repeat (SSR) anchor markers are highlighted in bold italics. 


\section{LG8 [1]}

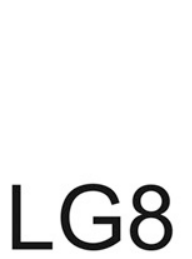

LG8 [2]

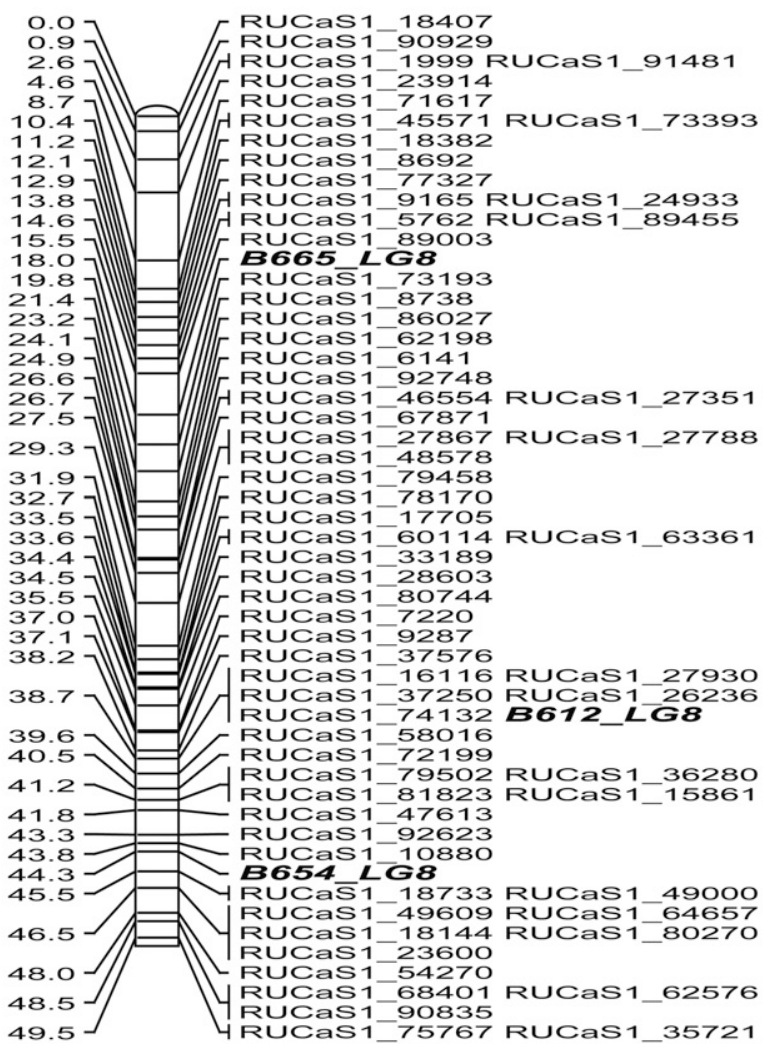

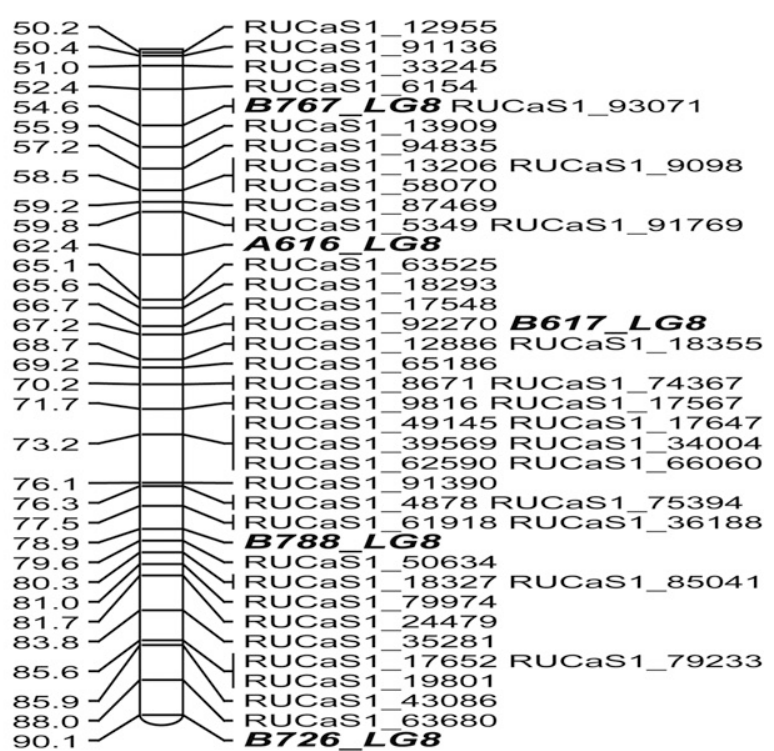

Supplemental Fig. 8. Representation of european hazelnut linkage group (LG) 8. Microsatellite or simple sequence repeat (SSR) anchor markers are highlighted in bold italics. 

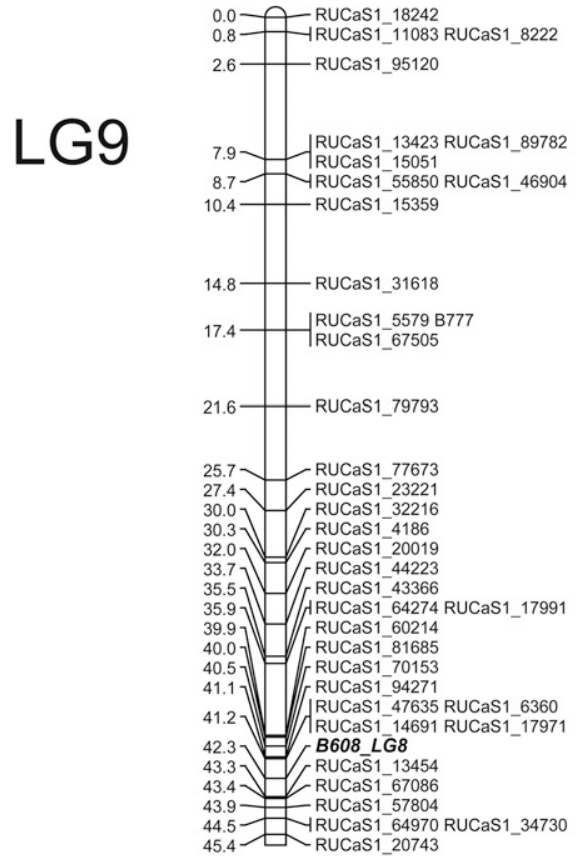

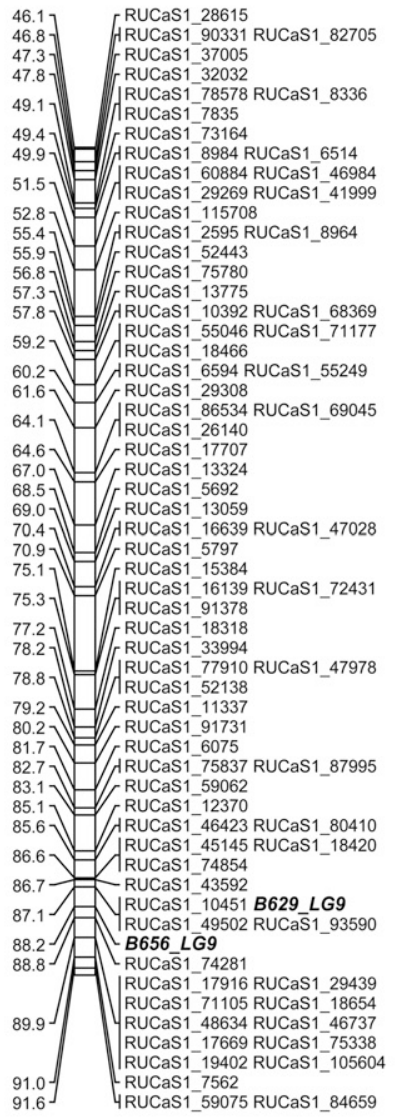

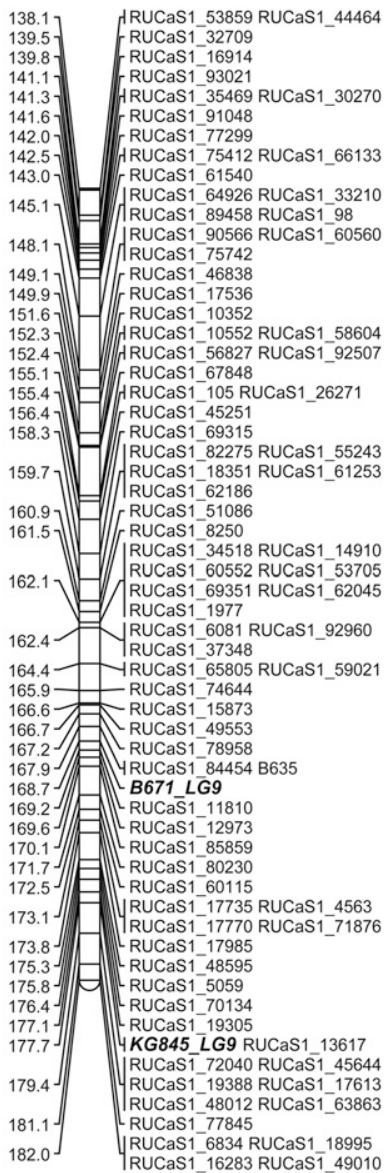

Supplemental Fig. 9. Representation of european hazelnut linkage group (LG) 9. Microsatellite or simple sequence repeat (SSR) anchor markers are highlighted in bold italics. 


\section{LG10 [1]}

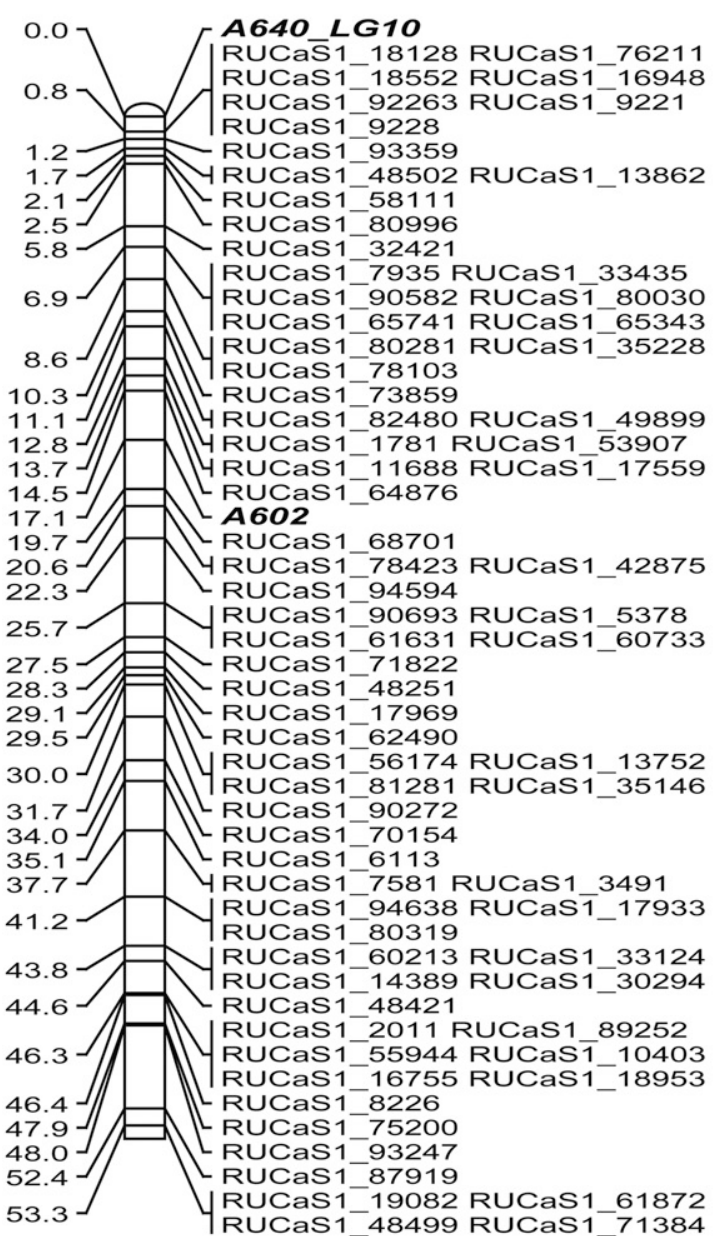

LG10 [2]

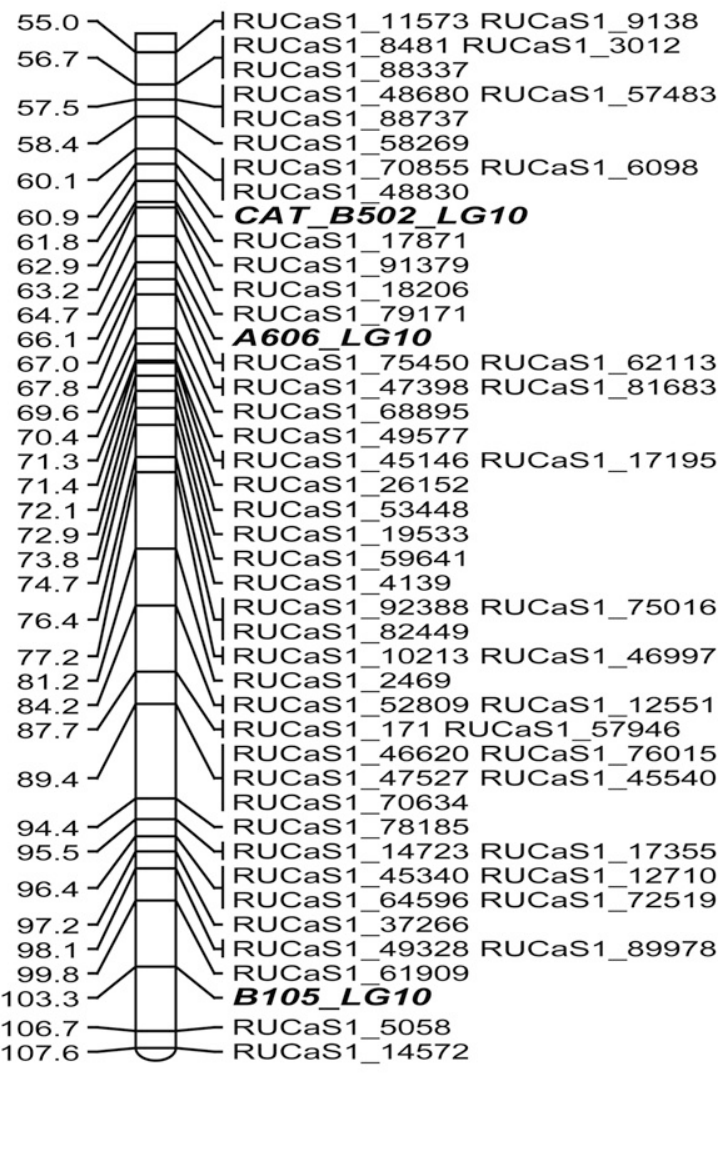

Supplemental Fig. 10. Representation of european hazelnut linkage group (LG) 10. Microsatellite or simple sequence repeat (SSR) anchor markers are highlighted in bold italics. 

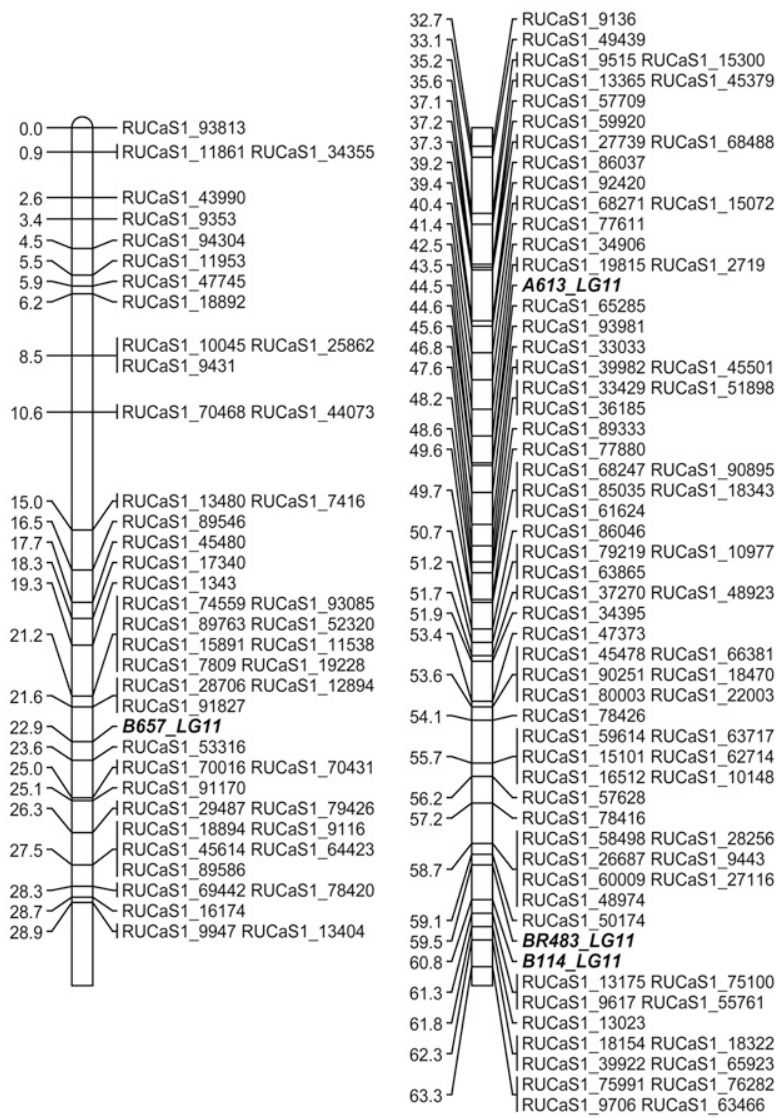

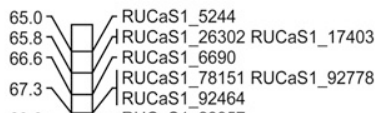

RUCaS1_92464

71.5 RUCaS1 93620 RUCaS1 48276

71.7 - RUCaS1_10606

|RUCaS1_6438 RUCaS1 14636

71.9 RUCaS1 89921 RUCaS1 74705

RUCaS1_63214 RUCaS1_76591

73.1-RUCaS1_115

73.7 RUCaS1_12537 RUCaS1_16259

73.7 RUCaS1_10896 RUCaS1_90121

FuCas1_19640 RUCas1_63149

7RUCaS1 1 73899

75.8 RUCaS1_17116

GB867_LG11 RUCaS1_14873

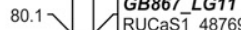

81.2 JRUCaS1-33262 RUCaS1 45410

81.8 2 RUCaS1_78046 RUCaS1_47808

RUCaS1_66856

RuCaS1-16231

84.8_RUCas1-46000

86.2 RUCaS1 56024 RUCaS1 45587

RUCaS1 58275

87.4 RUCaS1_1336 RUCaS1_46815

RUCaS1_70889

89.2 RUCaS1_48297

91.1_JRUCaS1_82320 RUCaS1_33538

93.0] RUCaS1_50490

RUCas1_48798 RUCaS1_10952

93.5

RUCaS1_8785 RUCaS1_89988

$94.3 \longrightarrow$ RUCaS1 6284

95.2- $\quad$ RUCaS1_81199 RUCaS1_66895

A RUCaS1_57531 RUCaS1_3710

95.7 _RUCasi_9569

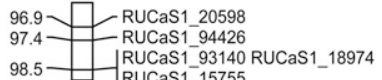

98.5- RUCaS1 193140

98.9 RUCaS1-90823

RUCaS1_27507 RUCaS1_30923

RUCaS1_10713 RUCaS1_28023

102.9 1 RUCaS1_88190

$104.0 \quad-\quad$ RUCaS1 93511 RUCaS1 64289

$105.1-1$ RUCaS1_133 RUCaS1 84153

$105.7-\quad-\quad$ RUCaS1_11885 RUCas̄i_72007

106.6-RUCaS1_35178

106.8 $\mathrm{RUC}$

107.6 RUCaS1_56622

$109.6-J R U C a S 111935$ RUCaS1 63576

109.9 RUCaS1_14133

$111.7-\longrightarrow$ RUCaS1 14698 RUCaS1 59638

$112.3-$ RUCaS1 64325

113.5- RUCaS1_90071

114.7 RUCaS1_10062

115.6 _RUCaS1_10485 RUCaS1_69429

115.6 RUCaS1_10485 RUCaS1_69429

$117.1-120 \mathrm{C}$

117.7 IRUCaS1_36403 RUCaS1 64955

$119.7-$ RUCaS1_73304

121.0_ RUCaS1 49733

RUCas1 19966

122.3-_RUCaS1_93680 RUCaS1_74226

$123.6-[$ RUCaS1_17992 RUCaS1_63441

124.9- B106_LG11

Supplemental Fig. 11. Representation of european hazelnut linkage group (LG) 11. Microsatellite or simple sequence repeat (SSR) anchor markers are highlighted in bold italics. 